Article

\title{
Analytical Analysis of Heat Transfer and Entropy Generation in a Tube Filled with Double-Layer Porous Media
}

\author{
Kun Yang, Wei Huang, Xin Li and Jiabing Wang *
}

School of Energy and Power Engineering, Huazhong University of Science and Technology, Wuhan 430074, China; yangk@hust.edu.cn (K.Y.); 13035115213@163.com (W.H.); m201971199@hust.edu.cn (X.L.)

* Correspondence: wjb@hust.edu.cn; Tel.: +86-027-87542517

Received: 13 September 2020; Accepted: 23 October 2020; Published: 26 October 2020

check for updates

\begin{abstract}
The heat transfer and entropy generation in a tube filled with double-layer porous media are analytically investigated. The wall of the tube is subjected to a constant heat flux. The Darcy-Brinkman model is utilized to describe the fluid flow, and the local thermal non-equilibrium model is employed to establish the energy equations. The solutions of the temperature and velocity distributions are analytically derived and validated in limiting case. The analytical solutions of the local and total entropy generation, as well as the Nusselt number, are further derived to analyze the performance of heat transfer and irreversibility of the tube. The influences of the Darcy number, the Biot number, the dimensionless interfacial radius, and the thermal conductivity ratio, on flow and heat transfer are discussed. The results indicate, for the first time, that the Nusselt number for the tube filled with double-layer porous media can be larger than that for the tube filled with single layer porous medium, while the total entropy generation rate for the tube filled with double-layer porous media can be less than that for the tube filled with single layer porous medium. And the dimensionless interfacial radius corresponding to the maximum value of the Nusselt number is different from that corresponding to the minimum value of the total entropy generation rate.
\end{abstract}

Keywords: porous media; double-layer; local thermal non-equilibrium; analytical solution

\section{Introduction}

Porous media has considerable advantages of large specific surface area and complex pore structure, which makes the porous media have excellent heat transfer performance and extensive range of industrial applications, such as sewage treatment, electronic device cooling, fuel cells, solar collectors, compact heat exchanger, and heat transfer enhancement.

Heat transfer and transport phenomenon in porous media has gained increasing attention. Kim et al. [1] derived the analytical solution for temperature field in the microchannel heat sink. Jing et al. [2,3] theoretically and numerically studied the flow and heat transfer in tree-like branching microchannel. Pavel and Mohamad [4] conducted experimental work to investigate the influence of inserting porous media on heat transfer rate within a tube. Lu et al. [5] theoretically study the effects of porosity and pore size on heat transfer in a pipe filled with high porosity porous media. Yang and Vafai [6,7] analyzed the phenomenon of heat flux bifurcation in a channel filled with porous media. Xu et al. [8] studied the forced convection in a porous parallel-plate channel using a modified fin analysis method. Dehghan et al. [9] investigated the developed region in a porous tube using perturbation techniques to study the influence of local thermal non-equilibrium (LTNE) condition. Zhang et al. [10] semi-analytically studied the flow and heat transfer in a porous tube with the inertia term, thermal dispersion and axial conduction ignored, and numerically analyzed the influences of 
the neglected terms. Dehghan et al. [11] studied the influences of the thermal conductivity varying with temperature on heat transfer in a porous parallel-plate channel using perturbation methods. Yang et al. [12] analytically analyzed two approaches to describe the adiabatic boundary condition and the heat flux bifurcation phenomenon for a porous parallel-plate channel. Torabi et al. [13-15] and Yang et al. [16] investigated the heat transfer and irreversibility in a parallel-plate channel partially filled with single-layer porous media and N-layer porous medium, respectively. Yang et al. [17] and Mahmoudi et al. [18] also investigated the heat transfer in a channel partially filled with porous media. Mohamed et al. [19] explored the influences of essential parameters on temperature profiles in a circular cylinder filled with porous media. Buonomo et al. [20] investigated the fully developed forced convection in parallel plates porous micro-channels. Based on the above studies, it can be concluded that porous media insert can effectively enhance heat transfer.

To analyze the effect of the heterogeneity of porous media on heat transfer, the channel filled with layered porous media was investigated. Nield and Kuznetsov [21] studied the influences of transverse variation of permeability and thermal conductivity on heat transfer in a channel filled with double-layer porous media based on Darcy equation and local thermal equilibrium (LTE) model. Nield and Kuznetsov extended their work to the case of asymmetric property variation and heating [22]. Nield et al. [23] and Sundaravadivelu et al. [24] investigated the influence of the viscosity varying with temperature on heat transfer in a channel filled with layered porous media. Nield and Kuznetsov [25] studied the interaction between the transverse heterogeneity of porous media and thermal development in a channel filled with layered porous media. Based on the above theoretical studies, the optimization of heat transfer performance in a channel filled with layered porous media was studied [26-29].

Most above-mentioned works for the channel filled with layered porous media were studied using Darcy equation and LTE model. However, the Darcy equation and LTE model neglect the viscous effect of impermeable wall and the heat exchange between two phases in porous media, respectively. To solve the problem, the Darcy-Brinkman equation and the local thermal non-equilibrium (LTNE) model can be used. Misra et al. [30] numerically studied the temperature distribution and entropy generation in a microfluidic tube using Darcy-Forchheimer-Brinkman equation. Nield and Kuznetsov analytically analyzed the effects of the transverse heterogeneity on heat transfer in a channel filled with layered porous media using Darcy-Brinkman equation [31] and LTNE model [32]. However, to the best of authors' knowledge, there are no related analytical works which utilized Darcy-Brinkman equation and LTNE model simultaneously published in the literature. Entropy generation is the key thermodynamic parameter to measure the irreversibility of the flow and heat transfer process. The smaller entropy generation within the system, the less energy is destructed [13-15]. However, there are few studies on the entropy generation in a compositive porous channel.

The main objective of this paper was to analytically investigate the heat transfer and entropy generation in a tube filled with double-layer porous media for the engineering applications including heat transfer enhancement and electronic device cooling. The constant heat flux boundary condition was applied on the wall of the tube. The Darcy-Brinkman equation and the LTNE model were adopted to establish momentum and energy equations. The analytical solutions of the temperature and velocity distributions were derived, and the analytical solutions of the Nusselt number, the local, and total entropy generation rate were further obtained. The influences of pertinent parameters were discussed. It was found for the first time that the performance of heat transfer for the tube filled with double-layer porous media can be better than that for the tube filled with single layer porous medium.

\section{Problem Descriptions}

\subsection{Physical Problem}

The schematic diagram of the tube studied in this study is shown in Figure 1. The radius of layer-1 is $r_{1}$, and the radius of tube is $r_{2}$. The wall of the tube is subjected to a constant heat flux, $q_{w}$. Due to 
the symmetry, only half of the tube is considered in this work. The following assumptions are invoked in analyzing this problem:

Each porous layer is homogenous and isotropic.

The flow and heat transfer are steady and fully developed.

The pertinent physical properties are constant.

The gravity is neglected.

The no-slip condition is used for the velocity boundary condition.

The internal heat source in the energy equation is not considered.

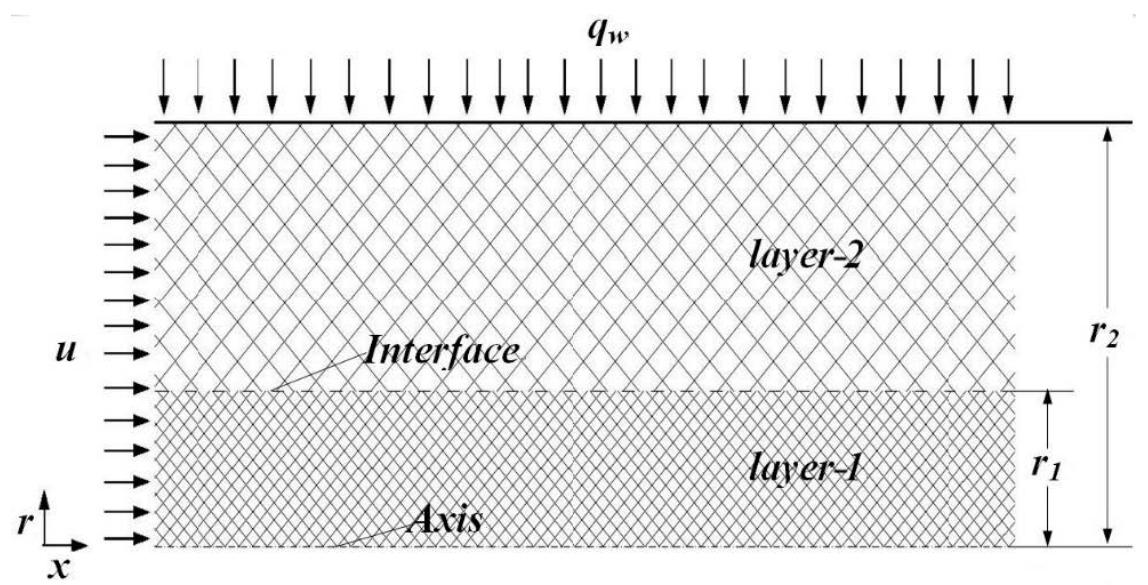

Figure 1. Geometry diagram of the tube filled with double-layer porous media and the corresponding coordinate system.

\subsection{Governing Equations}

Based on the above assumptions, the governing equations of the fluid flow and heat transfer are obtained.

The momentum equations are described by the Darcy-Brinkman model [33],

$$
\begin{aligned}
& \text { layer-1: }-\frac{d p}{d x}+\frac{\mu_{f}}{\varepsilon_{1}} \frac{1}{r} \frac{\partial}{\partial r}\left(r \frac{\partial u}{\partial r}\right)-\frac{\mu_{f}}{K_{1}} u=0\left(0<r<r_{1}\right), \\
& \text { layer-2: }-\frac{d p}{d x}+\frac{\mu_{f}}{\varepsilon_{2}} \frac{1}{r} \frac{\partial}{\partial r}\left(r \frac{\partial u}{\partial r}\right)-\frac{\mu_{f}}{K_{2}} u=0\left(r_{1}<r<r_{2}\right) .
\end{aligned}
$$

Energy equations of fluid phase are as follows [33]:

$$
\begin{aligned}
& \text { layer-1: } k_{f e 1}\left(\frac{\partial^{2} T_{f}}{\partial r^{2}}+\frac{1}{r} \frac{\partial T_{f}}{\partial r}\right)+h_{1} a_{1}\left(T_{s}-T_{f}\right)=\rho_{f} \mathcal{C}_{f} u \frac{\partial T_{f}}{\partial x}\left(0<r<r_{1}\right), \\
& \text { layer-2: } k_{f e 2}\left(\frac{\partial^{2} T_{f}}{\partial r^{2}}+\frac{1}{r} \frac{\partial T_{f}}{\partial r}\right)+h_{2} a_{2}\left(T_{s}-T_{f}\right)=\rho_{f} c_{f} u \frac{\partial T_{f}}{\partial x}\left(r_{1}<r<r_{2}\right) .
\end{aligned}
$$

Energy equations of solid phase are as follows [33]:

$$
\begin{aligned}
& \text { layer-1: } k_{s e 1}\left(\frac{\partial^{2} T_{s}}{\partial r^{2}}+\frac{1}{r} \frac{\partial T_{s}}{\partial r}\right)-h_{1} a_{1}\left(T_{s}-T_{f}\right)=0\left(0<r<r_{1}\right), \\
& \text { layer-2: } k_{s e 2}\left(\frac{\partial^{2} T_{s}}{\partial r^{2}}+\frac{1}{r} \frac{\partial T_{s}}{\partial r}\right)-h_{2} a_{2}\left(T_{s}-T_{f}\right)=0\left(r_{1}<r<r_{2}\right) .
\end{aligned}
$$


For thermally developed flow, the following equation is obtained based on energy balance within the tube.

$$
\frac{\partial T_{f}}{\partial x}=\frac{d T_{f b}}{d x}=\frac{2 q_{w}}{\rho_{f} \mathcal{C}_{f} u_{m} r_{2}} .
$$

By substituting Equation (7) into Equations (3) and (4), Equations (8) and (9) are obtained.

$$
\begin{aligned}
& \text { layer-1: } k_{f e 1}\left(\frac{\partial^{2} T_{f}}{\partial r^{2}}+\frac{1}{r} \frac{\partial T_{f}}{\partial r}\right)+h_{1} a_{1}\left(T_{s}-T_{f}\right)=\frac{2 q_{w}}{r_{2}} \frac{u}{u_{m}}\left(0<r<r_{1}\right), \\
& \text { layer-2: } k_{f e 2}\left(\frac{\partial^{2} T_{f}}{\partial r^{2}}+\frac{1}{r} \frac{\partial T_{f}}{\partial r}\right)+h_{2} a_{2}\left(T_{s}-T_{f}\right)=\frac{2 q_{w}}{r_{2}} \frac{u}{u_{m}}\left(r_{1}<r<r_{2}\right),
\end{aligned}
$$

where $u_{m}$ is written as

$$
u_{m}=\frac{1}{\pi r_{2}^{2}}\left(\int_{0}^{r_{1}} u_{1} \cdot 2 \pi r \cdot d r+\int_{r_{1}}^{r_{2}} u_{2} \cdot 2 \pi r \cdot d r\right)
$$

\subsection{Boundary Conditions}

The boundary condition at the wall for momentum equations and energy equations are represented as follows [5]:

$$
\begin{gathered}
u=0 \quad\left(r=r_{2}\right), \\
q_{w}=-k_{f e 2} \frac{\partial T_{f}}{\partial r}-k_{s e 2} \frac{\partial T_{s}}{\partial r} \quad T_{f}=T_{s}=T_{w} \quad\left(r=r_{2}\right) .
\end{gathered}
$$

Due to the symmetry configuration, the following boundary condition can be used at the axis of the tube.

$$
\frac{\partial u}{\partial r}=0 \quad \frac{\partial T_{s}}{\partial r}=\frac{\partial T_{f}}{\partial r}=0 \quad(r=0)
$$

At the interface between two layers porous media, the continuities of the velocity, shear stress, temperature of solid and fluid, heat flux of solid, and fluid are applied [16,34-36].

$$
u_{1}=u_{2} \frac{1}{\varepsilon_{1}} \frac{\partial u}{\partial r}=\frac{1}{\varepsilon_{2}} \frac{\partial u}{\partial r} T_{f 1}=T_{f 2} k_{f e 1} \frac{\partial T_{f}}{\partial r}=k_{f e 2} \frac{\partial T_{f}}{\partial r} T_{s 1}=T_{s 2} k_{s e 1} \frac{\partial T_{s}}{\partial r}=k_{s e 2} \frac{\partial T_{s}}{\partial r}\left(r=r_{1}\right)
$$

\subsection{Normalization}

The following dimensionless parameters are introduced to normalize the governing equations and boundary conditions:

$$
\begin{aligned}
& U=\frac{u}{u_{m}} \quad R=\frac{r}{r_{2}} \quad R_{1}=\frac{r_{1}}{r_{2}} \quad P=\frac{d p}{d x} \frac{r_{2}^{2}}{\mu_{f} u_{m}} \quad D a_{1}=\frac{K_{1}}{r_{2}^{2}} \quad D a_{2}=\frac{K_{2}}{r_{2}^{2}} \quad \theta_{s}=\frac{T_{s}-T_{w}}{q_{w w} r_{2} / k_{s e 1}} \\
& \theta_{f}=\frac{T_{f}-T_{w}}{q_{w w} r_{2} / k_{s e 1}} \quad B i_{1}=\frac{h_{1} a_{1} r_{2}^{2}}{k_{s e 1}} \quad B i_{2}=\frac{h_{2} a_{2} r_{2}^{2}}{k_{s e 1}} \quad k_{0}=\frac{k_{f}}{k_{s e 1}} \quad k_{1}=\frac{k_{f e 1}}{k_{s e 1}} \quad k_{2}=\frac{k_{f e 2}}{k_{s e 1}} \\
& k_{s 1}=1 \quad k_{s 2}=\frac{k_{s e 2}}{k_{s e 1}} \quad s_{1}=\sqrt{\frac{\varepsilon_{1}}{D a_{1}}} \quad s_{2}=\sqrt{\frac{\varepsilon_{2}}{D a_{2}}} \quad t_{1}=\sqrt{\frac{k_{1}+k_{s 1}}{k_{1} k_{s 1}} B i_{1}} \quad t_{2}=\sqrt{\frac{k_{2}+k_{s 2}}{k_{2} k_{s 2}} B i_{2}} \\
& F=\frac{T_{w} k_{s e 1}}{q_{w} r_{2}} \quad P e=\frac{c_{f} \rho_{f} r_{2} u_{m}}{2 k_{s e 1}} \quad B r=\frac{\mu_{f} u_{m}^{2}}{q_{w} r_{2}}
\end{aligned}
$$

The momentum Equations (1) and (2) are rewritten as

$$
\text { layer-1: } \frac{\partial^{2} U}{\partial R^{2}}+\frac{1}{R} \frac{\partial U}{\partial R}=\varepsilon_{1}\left(P+\frac{U}{D a_{1}}\right) \quad\left(0<R<R_{1}\right)
$$




$$
\text { layer-2: } \frac{\partial^{2} U}{\partial R^{2}}+\frac{1}{R} \frac{\partial U}{\partial R}=\varepsilon_{2}\left(P+\frac{U}{D a_{2}}\right) \quad\left(R_{1}<R<1\right) .
$$

The energy Equations (5), (6), (8), and (9) are rewritten as

$$
\begin{array}{ll}
\text { layer-1: } k_{s 1}\left(\frac{\partial^{2} \theta_{s}}{\partial R^{2}}+\frac{1}{R} \frac{\partial \theta_{s}}{\partial R}\right)-B i_{1}\left(\theta_{s}-\theta_{f}\right)=0 & \left(0<R<R_{1}\right), \\
\text { layer-2: } k_{s 2}\left(\frac{\partial^{2} \theta_{s}}{\partial R^{2}}+\frac{1}{R} \frac{\partial \theta_{s}}{\partial R}\right)-B i_{2}\left(\theta_{s}-\theta_{f}\right)=0 & \left(R_{1}<R<1\right), \\
\text { layer-1: } k_{1}\left(\frac{\partial^{2} \theta_{f}}{\partial R^{2}}+\frac{1}{R} \frac{\partial \theta_{f}}{\partial R}\right)+B i_{1}\left(\theta_{s}-\theta_{f}\right)=2 U & \left(0<R<R_{1}\right), \\
\text { layer-2: } k_{2}\left(\frac{\partial^{2} \theta_{f}}{\partial R^{2}}+\frac{1}{R} \frac{\partial \theta_{f}}{\partial R}\right)+B i_{2}\left(\theta_{s}-\theta_{f}\right)=2 U \quad\left(R_{1}<R<1\right) .
\end{array}
$$

Further, the boundary conditions are as follows,

$$
\begin{gathered}
R=1: U=0 \quad \theta_{f}=\theta_{s}=0, \\
R=0: \frac{\partial U}{\partial R}=0 \frac{\partial \theta_{s}}{\partial R}=\frac{\partial \theta_{f}}{\partial R}=0, \\
R=R_{1}: U_{1}=U_{2} \quad \frac{1}{\varepsilon_{1}} \frac{\partial U}{\partial R}=\frac{1}{\varepsilon_{2}} \frac{\partial U}{\partial R} \quad \theta_{f 1}=\theta_{f 2} \quad k_{1} \frac{\partial \theta_{f}}{\partial R}=k_{2} \frac{\partial \theta_{f}}{\partial R} \quad \theta_{s 1}=\theta_{s 2} \\
k_{s 1} \frac{\partial \theta_{s}}{\partial R}=k_{s 2} \frac{\partial \theta_{s}}{\partial R}
\end{gathered}
$$

\section{Analytical Solutions}

Equations (16)-(21) are solved by using Modified Bessel Equation (MBE) [37]. The solutions are shown as follows.

\subsection{Velocity Solutions}

Combining Equations (16) and (17) with boundary conditions Equations (22)-(24), the velocity field is obtained as follows:

$$
U=\left\{\begin{array}{ll}
P\left[C_{1} I_{0}\left(s_{1} R\right)+C_{2} K_{0}\left(s_{1} R\right)-D a_{1}\right] & \left(0<R<R_{1}\right) \\
P\left[C_{3} I_{0}\left(s_{2} R\right)+C_{4} K_{0}\left(s_{2} R\right)-D a_{2}\right] & \left(R_{1}<R<1\right)
\end{array},\right.
$$

where the functions $I_{0}(z)$ and $K_{0}(z)$ are the first and second kinds of zero order modified Bessel function.

$$
\begin{gathered}
\frac{1}{A} \int_{A} U d A=1, \\
P=\frac{1}{\left\{\frac{2 C_{1}}{s_{1}} I_{1}\left(s_{1} R_{1}\right) R_{1}-D a R_{1}^{2}+\frac{2 C_{3}}{s_{2}}\left[I_{1}\left(s_{2}\right)-I_{1}\left(s_{2} R_{1}\right) R_{1}\right]-\frac{2 C_{4}}{s_{2}}\left[K_{1}\left(s_{2}\right)-K_{1}\left(s_{2} R_{1}\right) R_{1}\right]-D a_{2}\left(1-R_{1}^{2}\right)\right\}}
\end{gathered}
$$

where the constants $C_{1}, C_{2}, C_{3}$, and $C_{4}$ are derived as follows:

$$
\begin{gathered}
C_{1}=A-B C_{4}, \\
C_{2}=0 \\
C_{3}=\frac{D a_{2}-C_{4} K_{0}\left(s_{2}\right)}{I_{0}\left(s_{2}\right)}
\end{gathered}
$$




$$
C_{4}=\frac{A I_{0}\left(s_{1} R_{1}\right)-D a_{1}+D a_{2}-\frac{I_{0}\left(s_{2} R_{1}\right)}{I_{0}\left(s_{2}\right)} D a_{2}}{\frac{K_{0}\left(s_{2} R_{1}\right) I_{0}\left(s_{2}\right)-K_{0}\left(s_{2}\right) I_{0}\left(s_{2} R_{1}\right)}{I_{0}\left(s_{2}\right)}+B I_{0}\left(s_{1} R_{1}\right)},
$$

where $A=\frac{\varepsilon_{1} s_{2}}{\varepsilon_{2} s_{1}} \cdot \frac{1}{I_{1}\left(s_{1} R_{1}\right)} \cdot \frac{I_{1}\left(s_{2} R_{1}\right)}{I_{0}\left(s_{2}\right)} D a_{2}$, and $B=\frac{\varepsilon_{1} s_{2}}{\varepsilon_{2} s_{1}} \cdot \frac{1}{I_{1}\left(s_{1} R_{1}\right)} \cdot \frac{K_{0}\left(s_{2}\right) I_{1}\left(s_{2} R_{1}\right)+K_{1}\left(s_{2} R_{1}\right) I_{0}\left(s_{2}\right)}{I_{0}\left(s_{2}\right)}$.

\subsection{Temperature Distribution}

The analytical solutions for temperature distributions are derived as follows: layer-1 $\left(0<R<R_{1}\right)$

$$
\begin{gathered}
\theta_{s}=\frac{P}{k_{s 1}+k_{1}}\left[\begin{array}{c}
D_{1}+D_{2} \operatorname{In} R+D_{3} I_{0}\left(t_{1} R\right)+D_{4} K_{0}\left(t_{1} R\right)- \\
\frac{2 t_{1}^{2}}{s_{1}^{2}\left(s_{1}^{2}-t_{1}^{2}\right)} C_{1} I_{0}\left(s_{1} R\right)-\frac{D a_{1}}{2}\left(R^{2}+\frac{4}{t_{1}^{2}}\right)
\end{array}\right], \\
\theta_{f}=\theta_{s}-\frac{P}{k_{1}}\left[D_{3} I_{0}\left(t_{1} R\right)+D_{4} K_{0}\left(t_{1} R\right)-\frac{2}{s_{1}^{2}-t_{1}^{2}} C_{1} I_{0}\left(s_{1} R\right)-\frac{2 D a_{1}}{t_{1}^{2}}\right],
\end{gathered}
$$

layer-2 $\left(R_{1}<R<1\right)$

$$
\begin{aligned}
& \theta_{s}=\frac{P}{k_{s 2}+k_{2}}\left\{\begin{array}{c}
D_{5}+D_{6} I n R+D_{7} I_{0}\left(t_{2} R\right)+D_{8} K_{0}\left(t_{2} R\right)- \\
\frac{2 t_{2}^{2}}{s_{2}^{2}\left(s_{2}^{2}-t_{2}^{2}\right)}\left[C_{3} I_{0}\left(s_{2} R\right)+C_{4} K_{0}\left(s_{2} R\right)\right]-\frac{D a_{2}}{2}\left(R^{2}+\frac{4}{t_{2}^{2}}\right)
\end{array}\right\}, \\
& \theta_{f}=\theta_{s}-\frac{P}{k_{2}}\left\{D_{7} I_{0}\left(t_{2} R\right)+D_{8} K_{0}\left(t_{2} R\right)-\frac{2}{s_{2}^{2}-t_{2}^{2}}\left[C_{3} I_{0}\left(s_{2} R\right)+C_{4} K_{0}\left(s_{2} R\right)\right]-\frac{2 D a_{2}}{t_{2}}\right\},
\end{aligned}
$$

where the constants $D_{1}-D_{8}$ are as follows:

$$
\begin{gathered}
D_{1}=w_{7} D_{8}+w_{8}, \\
D_{2}=0 \\
D_{3}=w_{2} D_{8}+w_{3}, \\
D_{4}=0 \\
D_{5}=w_{11} \\
D_{6}=\frac{w_{5} D_{8}+w_{6}}{w_{4}}, \\
D_{7}=\frac{w_{1}-D_{8} K_{0}\left(t_{2}\right)}{I_{0}\left(t_{2}\right)}, \\
D_{8}=w_{10} / w_{9} .
\end{gathered}
$$

The constants $w_{1}-w_{11}$ are represented as follows:

$$
\begin{gathered}
w_{1}=\frac{2}{s_{2}^{2}-t_{2}^{2}}\left[C_{3} I_{0}\left(s_{2}\right)+C_{4} K_{0}\left(s_{2}\right)\right]+\frac{2 D a_{2}}{t_{2}^{2}} \\
w_{2}=\frac{k_{1}}{k_{2}} \frac{I_{0}\left(t_{2}\right) K_{0}\left(t_{2} R_{1}\right)-I_{0}\left(t_{2} R_{1}\right) K_{0}\left(t_{2}\right)}{I_{0}\left(t_{2}\right) I_{0}\left(t_{1} R_{1}\right)}, \\
w_{3}=\frac{k_{1}}{k_{2} I_{0}\left(t_{1} R_{1}\right)}\left\{\frac{I_{0}\left(t_{2} R_{1}\right)}{I_{0}\left(t_{2}\right)} w_{1}-\frac{2 D a_{2}}{t_{2}^{2}}-\frac{2}{s_{2}^{2}-t_{2}^{2}}\left[C_{3} I_{0}\left(s_{2} R_{1}\right)+C_{4} K_{0}\left(s_{2} R_{1}\right)\right]\right\} \\
+\frac{2 C_{1} I_{0}\left(s_{1} R_{1}\right)}{\left(s_{1}^{2}-t_{1}^{2}\right) I_{0}\left(t_{1} R_{1}\right)}+\frac{2 D a_{1}}{t_{1}^{2} I_{0}\left(t_{1} R_{1}\right)}
\end{gathered}
$$




$$
\begin{aligned}
& w_{4}=\frac{k_{s 2}}{\left(k_{2}+k_{s 2}\right) R_{1}}, \\
& w_{5}=\frac{k_{s 1} t_{1} I_{1}\left(t_{1} R_{1}\right) w_{2}}{k_{s 1}+k_{1}}+\frac{k_{s 2} t_{2}}{k_{s 2}+k_{2}} \cdot \frac{K_{0}\left(t_{2}\right) I_{1}\left(t_{2} R_{1}\right)+K_{1}\left(t_{2} R_{1}\right) I_{0}\left(t_{2}\right)}{I_{0}\left(t_{2}\right)}, \\
& w_{6}=\frac{k_{s 1} t_{1} I_{1}\left(t_{1} R_{1}\right) w_{3}}{k_{s 1}+k_{1}}-\frac{k_{s 1}}{k_{s 1}+k_{1}}\left[\frac{2 t_{1}^{2}}{s_{1}\left(s_{1}^{2}-t_{1}^{2}\right)} C_{1} I_{1}\left(s_{1} R_{1}\right)+D a_{1} R_{1}\right] \\
& -\frac{k_{s 2}}{k_{s 2}+k_{2}} \cdot\left\{\frac{I_{1}\left(t_{2} R_{1}\right) w_{1} t_{2}}{I_{0}\left(t_{2}\right)}-\frac{2 t_{2}^{2}}{s_{2}\left(s_{2}^{2}-t_{2}^{2}\right)}\left[C_{3} I_{1}\left(s_{1} R_{1}\right)-C_{4} K_{1}\left(s_{2} R_{1}\right)\right]-D a_{2} R_{1}\right\}, \\
& w_{7}=\frac{k_{1}+k_{s 1}}{k_{2}+k_{s 2}}\left[\frac{w_{5} \operatorname{In} R_{1}}{w_{4}}+\frac{I_{0}\left(t_{2}\right) K_{0}\left(t_{2} R_{1}\right)-K_{0}\left(t_{2}\right) I_{0}\left(t_{2} R_{1}\right)}{I_{0}\left(t_{2}\right)}\right]-w_{2} I_{0}\left(t_{1} R_{1}\right), \\
& w_{8}=\frac{k_{1}+k_{s 1}}{k_{2}+k_{s 2}}\left\{\begin{array}{l}
w_{11}+\frac{w_{6} I n R_{1}}{w_{4}}+\frac{I_{0}\left(t_{2} R_{1}\right) w_{1}}{I_{0}\left(t_{2}\right)}-\frac{2 t_{2}^{2}}{s_{2}^{2}\left(s_{2}^{2}-t_{2}^{2}\right)}\left[C_{3} I_{0}\left(s_{2} R_{1}\right)+C_{4} K_{0}\left(s_{2} R_{1}\right)\right] \\
-\frac{D a_{2}}{2}\left(R_{1}^{2}+\frac{4}{t_{2}^{2}}\right)
\end{array}\right\} \\
& -w_{3} I_{0}\left(t_{1} R_{1}\right)+\frac{2 t_{1}^{2}}{s_{1}^{2}\left(s_{1}^{2}-t_{1}^{2}\right)} C_{1} I_{0}\left(s_{1} R_{1}\right)+\frac{D a_{1}}{2}\left(R_{1}^{2}+\frac{4}{t_{1}^{2}}\right), \\
& w_{9}=\frac{k_{s 2} t_{2}}{k_{2}+k_{s 2}} \cdot \frac{K_{0}\left(t_{2}\right) I_{1}\left(t_{2} R_{1}\right)+I_{0}\left(t_{2}\right) K_{1}\left(t_{2} R_{1}\right)}{I_{0}\left(t_{2}\right)}+\frac{k_{2}}{k_{2}+k_{s 2}} \cdot \frac{w_{5}}{w_{4} R_{1}}+\frac{k_{s 1} t_{1} I_{1}\left(t_{1} R_{1}\right) w_{2}}{k_{1}+k_{s 1}}, \\
& w_{10}=\frac{2 s_{1} C_{1} I_{1}\left(s_{1} R_{1}\right)}{s_{1}^{2}-t_{1}^{2}}-\frac{k_{1}}{k_{1}+k_{s 1}}\left[\frac{2 t_{1}^{2}}{\left(s_{1}^{2}-t_{1}^{2}\right) s_{1}} C_{1} I_{1}\left(s_{1} R_{1}\right)+D a_{1} R_{1}\right]-\frac{k_{s 1} t_{1} I_{1}\left(t_{1} R_{1}\right) w_{3}}{k_{1}+k_{s 1}} \\
& -\frac{k_{2}}{k_{2}+k_{s 2}}\left\{\frac{w_{6}}{w_{4} R_{1}}-\frac{2 t_{2}^{2}}{s_{2}\left(s_{2}^{2}-t_{2}^{2}\right)}\left[C_{3} I_{1}\left(s_{2} R_{1}\right)-C_{4} K_{1}\left(s_{2} R_{1}\right)\right]-D a_{2} R_{1}\right\} \\
& -\frac{2 s_{2}}{s_{2}^{2}-t_{2}^{2}}\left[C_{3} I_{1}\left(s_{2} R_{1}\right)-C_{4} K_{1}\left(s_{2} R_{1}\right)\right]+\frac{k_{s 2} t_{2} I_{1}\left(t_{2} R_{1}\right) w_{1}}{\left(k_{2}+k_{s 2}\right) I_{0}\left(t_{2}\right)}, \\
& w_{11}=\frac{D a_{2}}{2}-\frac{2\left[C_{3} I_{0}\left(s_{2}\right)+C_{4} K_{0}\left(s_{2}\right)\right]}{s_{2}^{2}} .
\end{aligned}
$$

\subsection{Dimensionless Parameters}

Based on the analytical solutions for velocity field and temperature distribution, the friction coefficient and the Nuesselt number are obtained as follows:

$$
\begin{gathered}
f=\frac{-d p / d x \cdot 4 r_{2}}{\rho_{f} u_{m}^{2}}=-\frac{8 P}{D a \cdot \operatorname{Re}}, \\
N u=\frac{\bar{h}}{k_{f}} \cdot 2 R=\frac{2 R q_{w}}{k_{f}\left(T_{w}-T_{f . b}\right)}=-\frac{2}{k_{0} \cdot \theta_{f . b}},
\end{gathered}
$$

where $\theta_{f, b}$ can be deduced by Equation (57). The detail formula for $\theta_{f, b}$ is presented in the Appendix A.

$$
\theta_{f, b}=\frac{\frac{1}{A} \int_{A} U \theta_{f} d A}{\frac{1}{A} \int_{A} U d A}=\frac{1}{\pi} \int_{0}^{2 \pi} \int_{0}^{1} U \theta_{f} R d R d \varphi=2 \int_{0}^{R_{1}} U \theta_{f} R d R+2 \int_{R_{1}}^{1} U \theta_{f} R d R .
$$

\subsection{Entropy Generation Rate}

The irreversibility of the studied system can be related to two effects: heat transfer across a finite (nonzero) temperature difference, as well as fluid friction [38]. Based on the equation mentioned above, the detailed derivations of the local entropy generation rates within two phases of porous media are 
given as follows [14], respectively:

layer-1:

$$
\begin{gathered}
S_{f 1}^{\prime \prime \prime}=\frac{k_{f e 1}}{T_{f 1}^{2}}\left[\left(\frac{\partial T_{f 1}}{\partial x}\right)^{2}+\left(\frac{\partial T_{f 1}}{\partial r}\right)^{2}\right]+\frac{h_{1} a_{1}\left(T_{s 1}-T_{f 1}\right)^{2}}{T_{s 1} T_{f 1}}+\frac{\mu_{f} u_{f 1}^{2}}{K_{1} T_{f 1}}+\frac{\mu_{e f f}}{T_{f 1}}\left(\frac{\partial u_{f 1}}{\partial r}\right)^{2}\left(0<r<r_{1}\right) \\
S_{s 1}^{\prime \prime \prime}=\frac{k_{s e 1}}{T_{s 1}^{2}}\left[\left(\frac{\partial T_{s 1}}{\partial x}\right)^{2}+\left(\frac{\partial T_{s 1}}{\partial r}\right)^{2}\right]+\frac{h_{1} a_{1}\left(T_{s 1}-T_{f 1}\right)^{2}}{T_{s 1} T_{f 1}}\left(0<r<r_{1}\right),
\end{gathered}
$$

layer-2:

$$
\begin{gathered}
S_{f 2}^{\prime \prime \prime}=\frac{k_{f e 2}}{T_{f 2}^{2}}\left[\left(\frac{\partial T_{f 2}}{\partial x}\right)^{2}+\left(\frac{\partial T_{f 2}}{\partial r}\right)^{2}\right]+\frac{h_{2} a_{2}\left(T_{s 2}-T_{f 2}\right)^{2}}{T_{s 2} T_{f 2}}+\frac{\mu_{f} u_{f 2}^{2}}{K_{2} T_{f 2}}+\frac{\mu_{e f f}}{T_{f 2}}\left(\frac{\partial u_{f 2}}{\partial r}\right)^{2}\left(r_{1}<r<r_{2}\right) \\
S_{s 2}^{\prime \prime \prime}=\frac{k_{s e 2}}{T_{s 2}^{2}}\left[\left(\frac{\partial T_{s 2}}{\partial x}\right)^{2}+\left(\frac{\partial T_{s 2}}{\partial r}\right)^{2}\right]+\frac{h_{2} a_{2}\left(T_{s 2}-T_{f 2}\right)^{2}}{T_{s 2} T_{f 2}}\left(r_{1}<r<r_{2}\right)
\end{gathered}
$$

In terms of the dimensionless parameters shown in Equation (15), the dimensionless local entropy generation rates are defined,

layer-1:

$$
\begin{gathered}
N_{f 1}^{\prime \prime \prime}=\frac{S_{f 1}^{\prime \prime \prime} \cdot r_{2}^{2}}{k_{s e 1}}=\frac{k_{1}\left[\left(\frac{\partial \theta_{f 1}}{\partial R}\right)^{2}+\frac{1}{P e^{2}}\right]}{\left(\theta_{f 1}+F\right)^{2}}+\frac{B i_{1}\left(\theta_{s 1}-\theta_{f 1}\right)^{2}}{\left(\theta_{f 1}+F\right)\left(\theta_{s 1}+F\right)} \quad\left(0<R<R_{1}\right), \\
+\frac{B r \cdot U_{f 1}^{2}}{D a_{1}\left(\theta_{f 1}+F\right)}+\frac{B r}{\varepsilon_{1}\left(\theta_{f 1}+F\right)}\left(\frac{\partial U_{f 1}}{\partial R}\right)^{2} \\
N_{s 1}^{\prime \prime \prime}=\frac{S_{s 1}^{\prime \prime \prime} \cdot r_{2}^{2}}{k_{s e 1}}=\frac{\left[\left(\frac{\partial \theta_{s 1}}{\partial R}\right)^{2}+\frac{1}{P e^{2}}\right]}{\left(\theta_{s 1}+F\right)^{2}}+\frac{B i_{1}\left(\theta_{s 1}-\theta_{f 1}\right)^{2}}{\left(\theta_{f 1}+F\right)\left(\theta_{s 1}+F\right)} \quad\left(0<R<R_{1}\right),
\end{gathered}
$$

layer-2:

$$
\begin{gathered}
N_{f 2}^{\prime \prime \prime}=\frac{S_{f 2}^{\prime \prime \prime} \cdot r_{2}^{2}}{k_{s e 1}}=\frac{k_{2}\left[\left(\frac{\partial \theta_{f 2}}{\partial R}\right)^{2}+\frac{1}{P e^{2}}\right]}{\left(\theta_{f 2}+F\right)^{2}}+\frac{B i_{2}\left(\theta_{s 2}-\theta_{f 2}\right)^{2}}{\left(\theta_{f 2}+F\right)\left(\theta_{s 2}+F\right)} \quad\left(R_{1}<R<1\right), \\
+\frac{B r \cdot U_{f 2}^{2}}{D a_{2}\left(\theta_{f 2}+F\right)}+\frac{B r}{\varepsilon_{2}\left(\theta_{f 2}+F\right)}\left(\frac{\partial U_{f 2}}{\partial R}\right)^{2} \\
N_{s 2}^{\prime \prime \prime}=\frac{S_{s 2}^{\prime \prime \prime} \cdot r_{2}^{2}}{k_{s e 1}}=\frac{k_{s 2}\left[\left(\frac{\partial \theta_{s 2}}{\partial R}\right)^{2}+\frac{1}{P e^{2}}\right]}{\left(\theta_{s 2}+F\right)^{2}}+\frac{B i_{2}\left(\theta_{s 2}-\theta_{f 2}\right)^{2}}{\left(\theta_{f 2}+F\right)\left(\theta_{s 2}+F\right)} \quad\left(R_{1}<R<1\right) .
\end{gathered}
$$

Accordingly, the dimensionless total entropy generation rate for the tube can be calculated using Equation (66).

$$
N_{t}=2 \pi\left[\int_{0}^{R_{1}}\left(N_{f 1}^{\prime \prime \prime}+N_{s 1}^{\prime \prime \prime}\right) R d R+\int_{R_{1}}^{1}\left(N_{f 2}^{\prime \prime \prime}+N_{s 2}^{\prime \prime \prime}\right) R d R\right] .
$$

\section{Results and Discussion}

\subsection{Validation of Solutions}

The analytical solutions in this paper can be validated for a limiting case in which the tube is fully filled with single layer porous medium. By setting the same pertinent parameters for the two porous layers, the present temperature distributions are exactly the same as those of the previous work of 
Lu et al. [5], as shown in Figure 2. When $\varepsilon_{1}=\varepsilon_{2}=1$ and the Darcy number approaches infinity, the present solution of the Nusselt number is 4.365 , which is very close to the classical theoretical and experimental value for thermally fully developed clear flow. By setting $B i_{1}=B i_{2}=10,000$, and the pertinent parameters is the same as those of Reference [39], the present solutions of the thermally fully developed Nusselt number are almost the same as the previous numerical results obtained by Pavel and Mohamad [39] under the LTE condition, as shown in Table 1. Furthermore, the comparison between the Nusselt number of present study and the experimental and numerical results obtained by Pavel and Mohamad [39] is shown in Table 2. It is found that the Nusselt number of present study is less than the experimental and numerical values. The reason is as follows: the Nusselt number of present study is for the thermally fully developed flow; however, both the experimental and numerical results of the [39] are the average Nusselt number for the entire tube, including the thermally developing section and the thermally fully developed section.

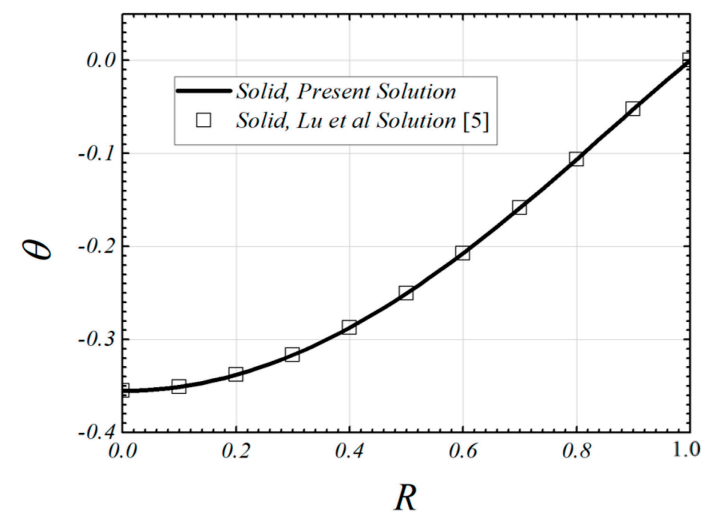

(a) solid phase

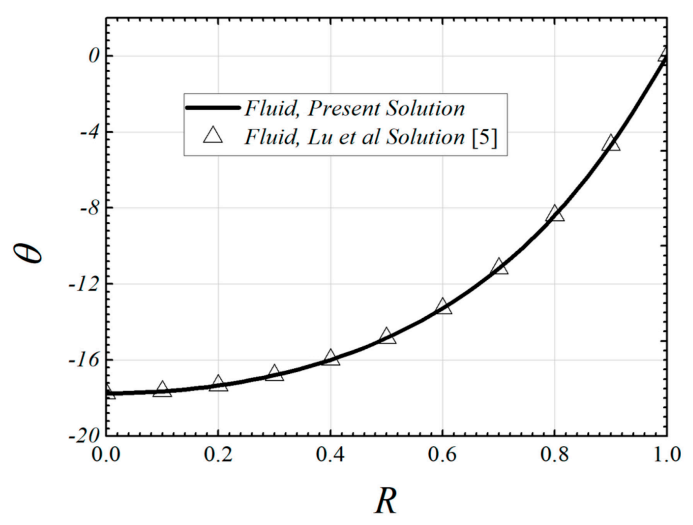

(b) fluid phase

Figure 2. Temperature distributions for the fluid and the solid phases of the present study and Reference [5]. $\left(\varepsilon_{1}=\varepsilon_{2}=0.9, D a_{1}=10^{-3}, D a_{2}=10^{-3}, B i_{1}=B i_{2}=0.1, k_{1}=k_{2}=0.01\right.$, $\left.R_{1}=0.5\right)$.

Table 1. The thermally fully developed $N u$ number of present study when $B i_{1}=B i_{2}=10,000$ and Reference [39].

\begin{tabular}{cccc}
\hline $\boldsymbol{D a}$ & $\frac{k_{f e}+k_{s e}}{k_{f}}$ & $\boldsymbol{N u}$ (Present Study) & $\boldsymbol{N u}([39])$ \\
\hline $10^{-2}$ & 1.0 & 5.9812 & 5.9848 \\
$10^{-3}$ & 1.0 & 7.1380 & 7.1360 \\
$10^{-4}$ & 1.0 & 7.6957 & 7.6955 \\
$10^{-5}$ & 1.0 & 7.9002 & 7.9012 \\
\hline
\end{tabular}

Table 2. The Nusselt number of the present study and the experimental and numerical average Nusselt number of Reference [39].

\begin{tabular}{cccccc}
\hline $\boldsymbol{\varepsilon}$ & $\boldsymbol{D} \boldsymbol{a}$ & $\frac{\boldsymbol{k}_{f e}+\boldsymbol{k}_{\text {se }}}{k_{f}}$ & $\begin{array}{c}\boldsymbol{N} \boldsymbol{u} \text { (Present Study When } \\
\left.\boldsymbol{B} \boldsymbol{i}_{1}=\mathbf{B} \boldsymbol{i}_{2}=\mathbf{1 0}, \mathbf{0 0 0}\right)\end{array}$ & $\begin{array}{c}\text { Experimental } \\
\text { Average } \boldsymbol{N} \boldsymbol{u}([39])\end{array}$ & $\begin{array}{c}\text { Numerical Average } \\
\boldsymbol{N} \boldsymbol{u}([39])\end{array}$ \\
\hline 0.993 & $6.1786 \times 10^{-4}$ & 1.06 & 7.7370 & 19.2204 & 21.8579 \\
0.981 & $1.9384 \times 10^{-4}$ & 1.5 & 11.3711 & 29.6612 & 34.0109 \\
\hline
\end{tabular}

\subsection{Velocity Distribution}

The influence of $D a_{1}$ on velocity profile is indicated in Figure 3a. As shown in the figure, the velocity profiles changes suddenly at the interface due to the sudden change of permeability across the interface. It is also found that the peak value of velocity appears in the layer with larger $D a$. In addition, when the $D a$ difference between two porous layers is large enough, the fluid within the 
layer with smaller $D a$ is almost stationary. This is because smaller $D a$ means lower permeability for the fluid flow. Therefore, the fluid tends to flow through the layer with larger $D a$, while only a small portion flows through the other layer at a lower velocity.

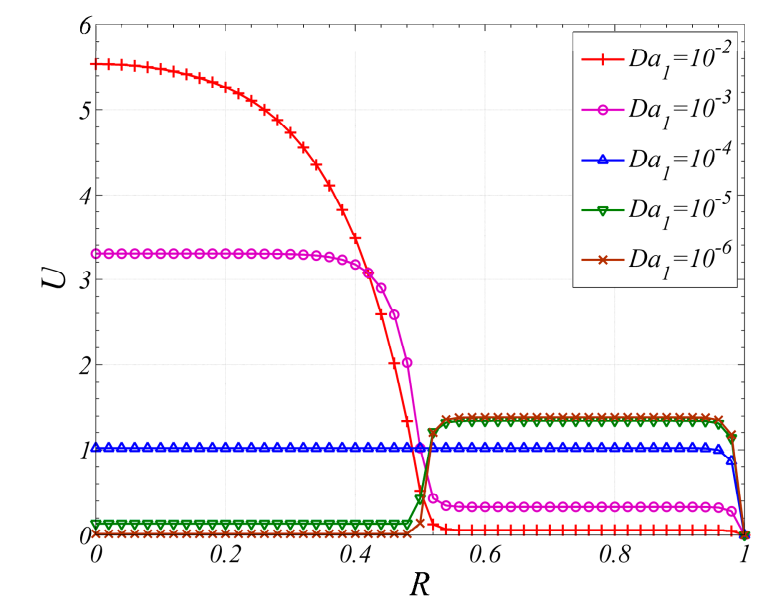

(a) $\varepsilon_{1}=\varepsilon_{2}=0.9, R_{1}=0.5, D a_{2}=10^{-4}$

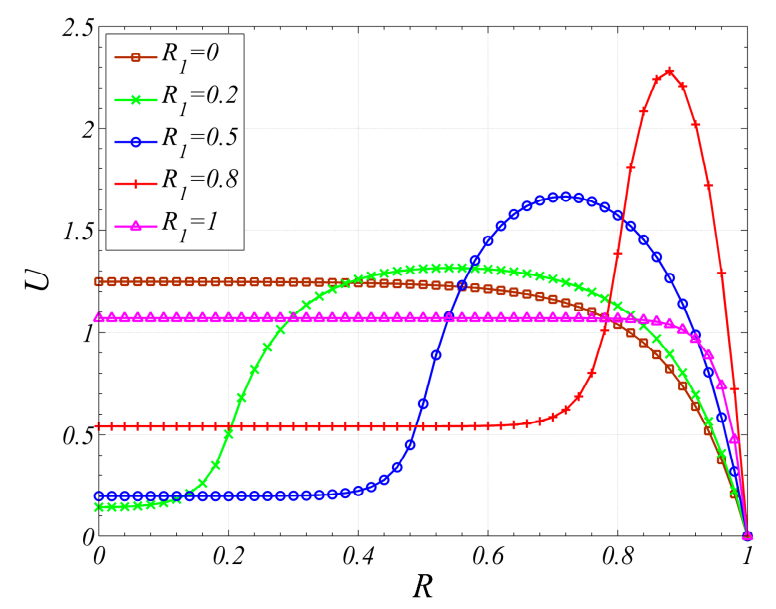

(b) $\varepsilon_{1}=\varepsilon_{2}=0.9, D a_{1}=10^{-3}, D a_{2}=10^{-2}$

Figure 3. Effects of pertinent parameters on velocity distribution.

Figure $3 \mathrm{~b}$ shows the velocity distribution for different dimensionless interfacial radius. As shown in the figure, for the case of when $D a_{1}<D a_{2}$ and $R_{1}<0.8$, the peak value of velocity increases and its location shifts towards the wall as dimensionless interfacial radius increases.

\subsection{Temperature Distribution}

The effect of $k_{s 2}$ on the temperature distributions is shown in Figure 4. As shown in the figure, both the temperatures of two phases increase with the increase of $k_{s 2}$.

The effects of $D a_{1}$ and $D a_{2}$ on the temperature distributions are shown in Figure 5. It is found that both increasing $D a_{2}$ and decreasing $D a_{1}$ can lead to more uniform temperature distribution of fluid phase. This is because, when large $D a_{2}$ or small $D a_{1}$ is employed, the majority of the fluid will flow through the layer-2, which is closer to heated wall. Therefore, more heat is transferred to fluid phase within the layer-2, and a small amount of energy is transferred into layer-1, which results in a small temperature difference within layer-1. 


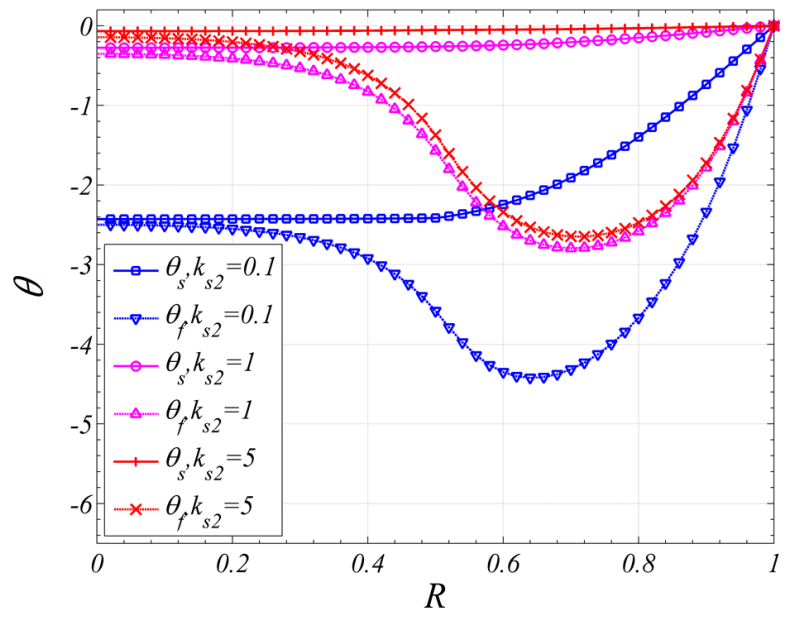

Figure 4. The effect of thermal conductivity ratio of solid on temperature distribution $\left(\varepsilon_{1}=\varepsilon_{2}=0.9\right.$, $\left.D a_{1}=10^{-5}, D a_{2}=10^{-3}, B i_{1}=B i_{2}=1, k_{1}=k_{2}=0.01, R_{1}=0.5\right)$.

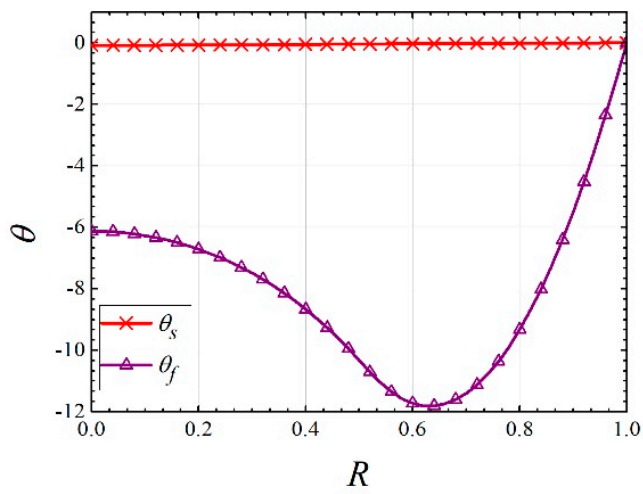

(a) $D a_{1}=10^{-5}, D a_{2}=10^{-3}$

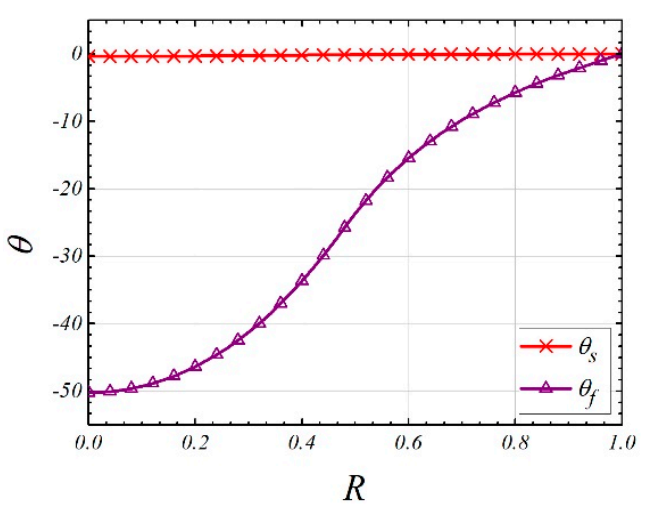

(c) $D a_{1}=10^{-3}, D a_{2}=10^{-5}$

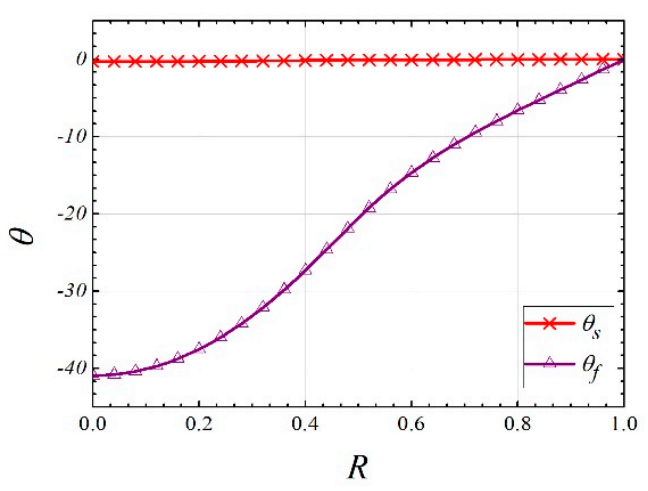

(b) $D a_{1}=10^{-2}, D a_{2}=10^{-3}$

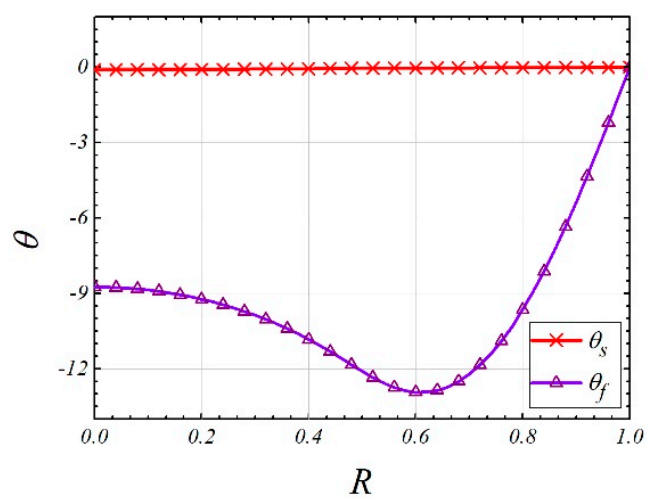

(d) $D a_{1}=10^{-3}, D a_{2}=10^{-2}$

Figure 5. The effects of $D a_{1}$ and $D a_{2}$ on the temperature distribution $\left(\varepsilon_{1}=\varepsilon_{2}=0.9, B i_{1}=B i_{2}=0.1\right.$, $\left.k_{1}=k_{2}=0.01, k_{s 2}=1, R_{1}=0.5\right)$.

\subsection{Heat Transfer Performance}

Figure 6 shows the trend of $N u$ versus $D a_{1}$. When the Biot number is small, which means a weak internal heat transfer between the solid and fluid phases in the porous media, the $\mathrm{Nu}$ decreases with the increase of the $D a_{1}$, as shown in Figure 6a. However, when the Biot number is large, the $N u$ increases firstly and then decreases gradually with the increase of the $D a_{1}$. As a result, a maximum value of $N u$ can be found in Figure 6b. 


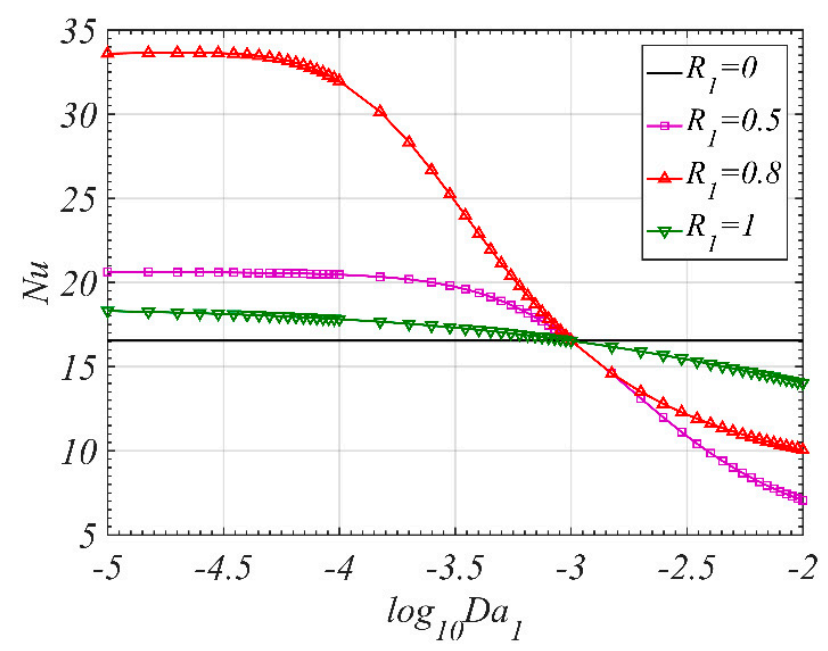

(a) $D a_{2}=10^{-3}, B i_{1}=B i_{2}=0.1$

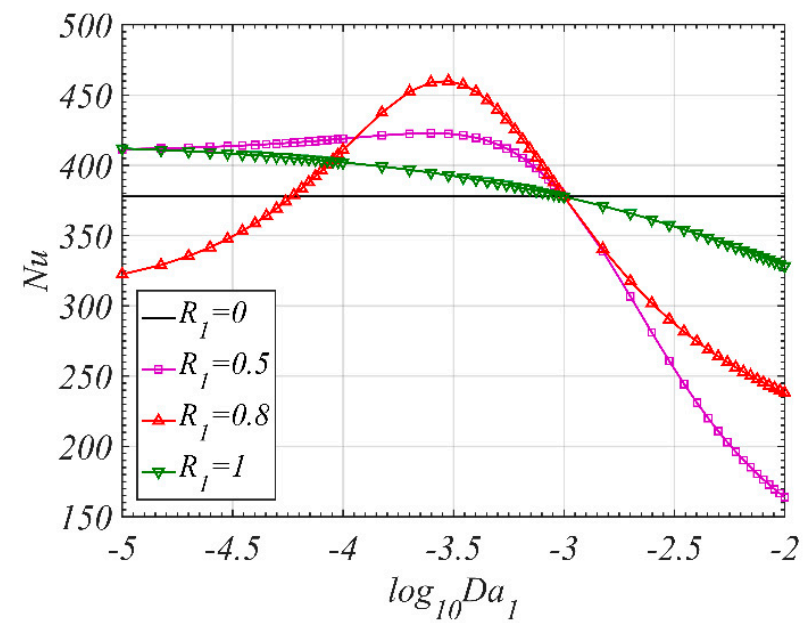

(b) $D a_{2}=10^{-3}, B i_{1}=B i_{2}=10$

Figure 6. The effect of $D a_{1}$ on $N u\left(\varepsilon_{1}=\varepsilon_{2}=0.9, k_{1}=k_{2}=0.01, k_{s 2}=1\right)$.

The effects of $R_{1}$ on $N u$ are shown in Figure 7. When $D a_{1}$ is larger than $D a_{2}$, the $N u$ decreases firstly and then increases with the increase of $R_{1}$, and a minimum value can be found, which is smaller than that for the tube fully filled with corresponding single layer porous medium $\left(R_{1}=0\right.$, or $\left.R_{1}=1\right)$. However, when $D a_{1}$ is smaller than $D a_{2}$, there is one local maximum value for the $N u$ when the Biot number is small, as shown in Figure 7a; or there are two local maximum values for the $N u$ when the Biot number is large, as shown in Figure $7 \mathrm{~b}$. It can be found that, when $D a_{1}$ is smaller than $D a_{2}$, the $N u$ for the tube filled with double-layer porous media can be larger than that for the tube filled with corresponding single layer porous medium $\left(R_{1}=0\right.$, or $\left.R_{1}=1\right)$. To the best of authors' knowledge, the above-mentioned analysis of the $N u$ for the tube filled with double-layer porous media is presented for the first time in the literature. 


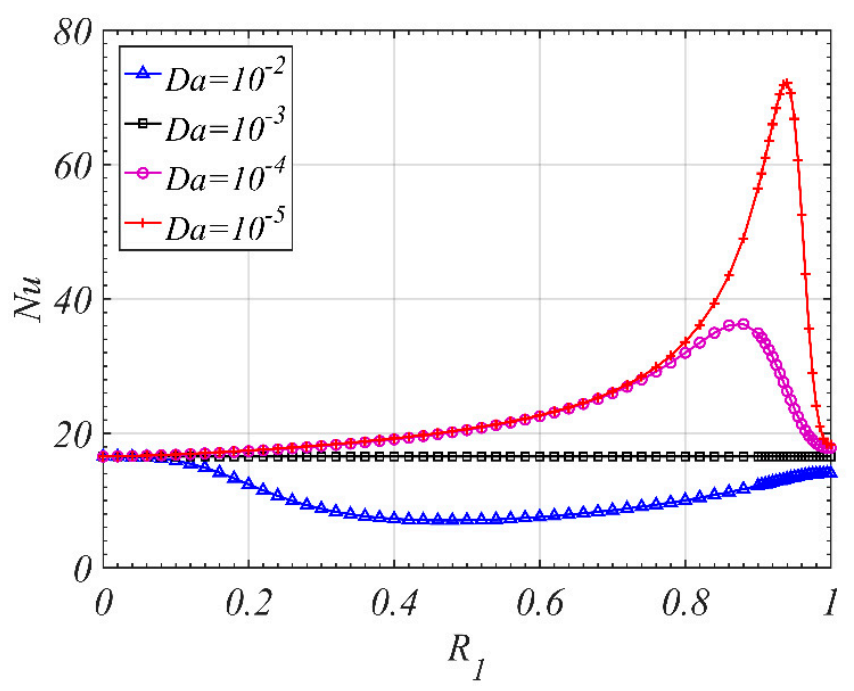

(a) $B i_{1}=B i_{2}=0.1$

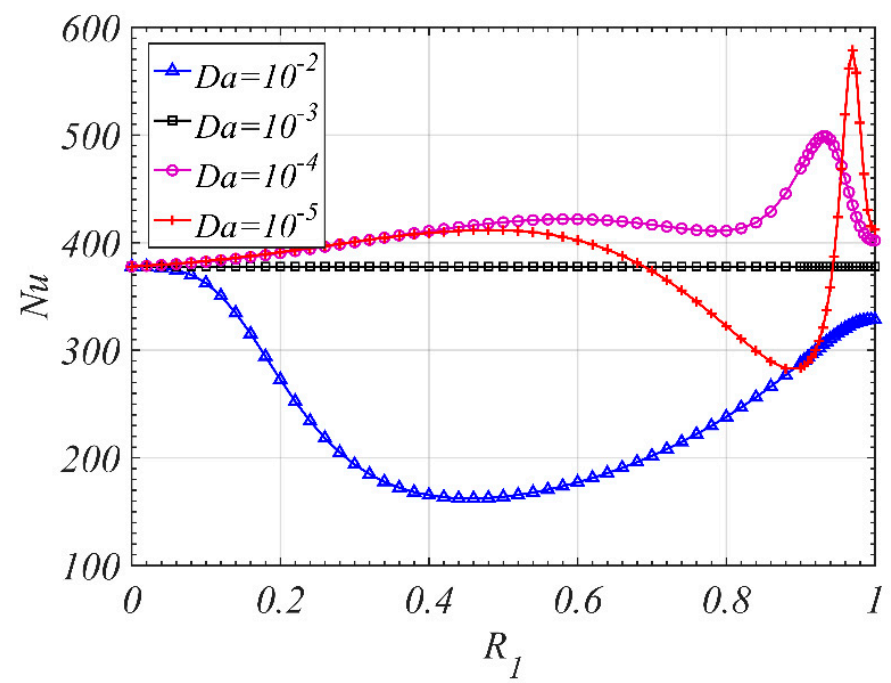

(b) $B i_{1}=B i_{2}=10$

Figure 7. $N u$ varies with $R_{1}$ for different $D a_{1}\left(\varepsilon_{1}=\varepsilon_{2}=0.9, k_{1}=k_{2}=0.01, k_{s 2}=1, D a_{2}=10^{-3}\right)$.

Figure 8 shows the trend of $N u$ versus $k_{s 2}$. When $k_{s 2}$ is less than one, the $N u$ increases dramatically with the increase of $k_{s 2}$. However, the ascending tendency become less obviously when $k_{s 2}>1$. This is because the value of the mean temperature of the fluid phase is negative, and it increases with the increase of $k_{s 2}$, which is more obvious when $k_{s 2}<1$, which can be found in Figure 4 . 


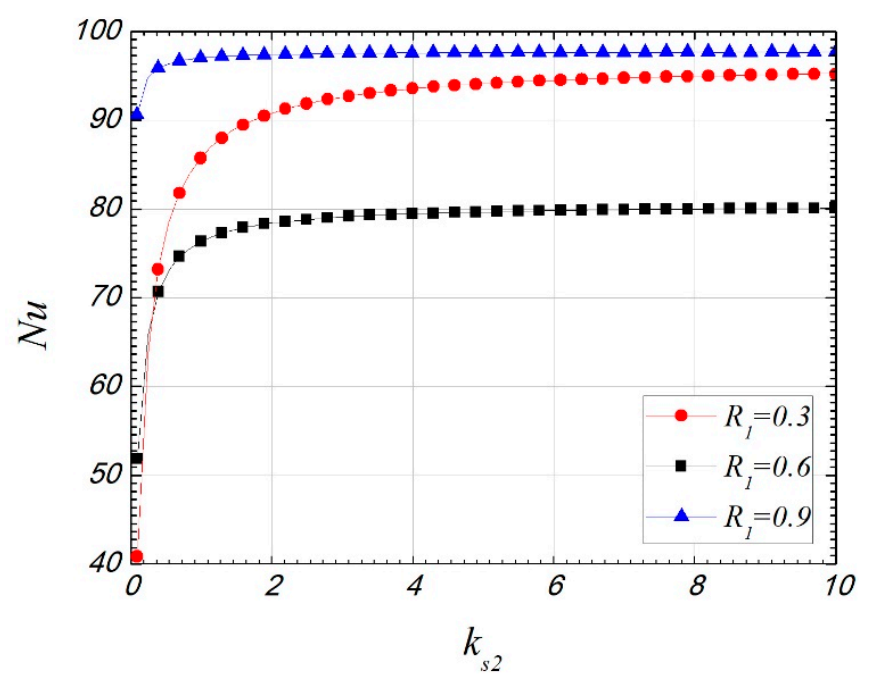

(a) $B i_{1}=B i_{2}=0.1$

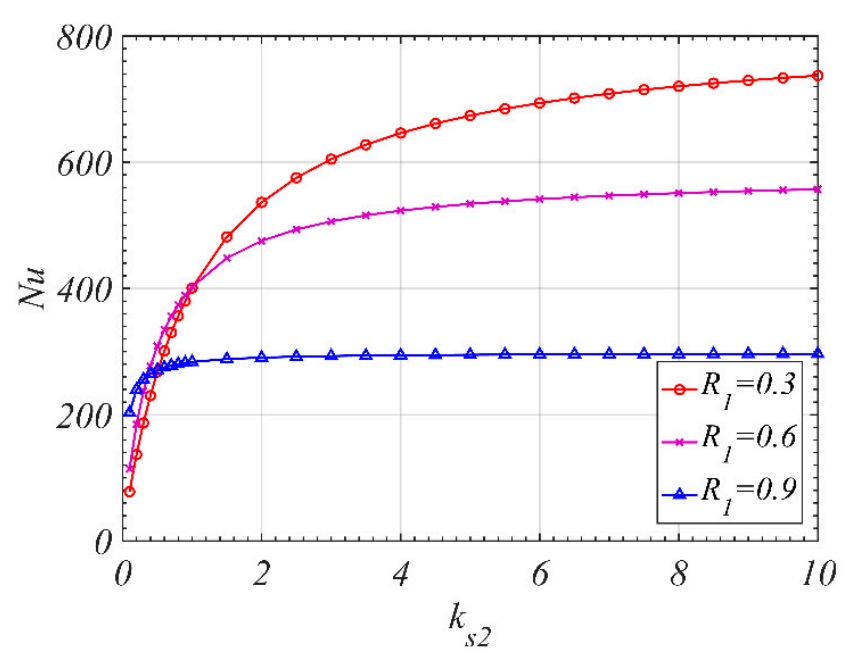

(b) $B i_{1}=B i_{2}=10$

Figure 8. $N u$ varies with $k_{s 2}\left(\varepsilon_{1}=\varepsilon_{2}=0.9, k_{1}=k_{2}=0.01, D a_{1}=10^{-5}, D a_{2}=10^{-3}\right)$.

\subsection{Local and Total Entropy Generation Rate}

The local and total entropy generation rates are used to describe irreversibility of the tube filled with porous media in this work. Figure 9 shows the changes of the dimensionless local entropy generation rate for four filling methods, including: (a) filled with double-layer porous media and $D a_{1}>D a_{2}$, (b) filled with double-layer porous media and $D a_{1}<D a_{2}$, (c) filled with single layer porous medium with small Darcy number, and (d) filled with single layer porous medium with large Darcy number. Among the four filling methods, filling the tube with double-layer porous media with smaller Darcy number for layer-1 (method b) can be more conducive to reduce the local entropy generation rate. 


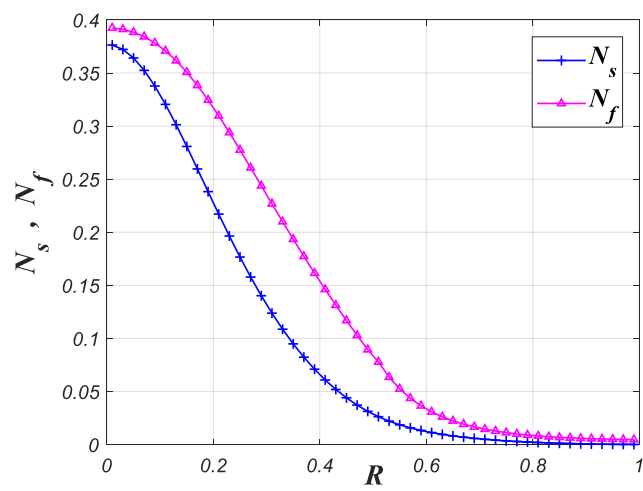

(a) Double-layer: $D a_{1}=10^{-2}, D a_{2}=10^{-3}$

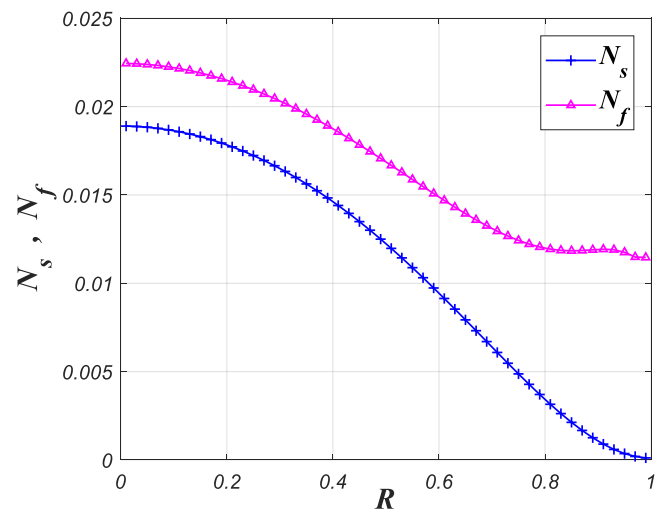

(c) Single layer: $D a=10^{-3}$

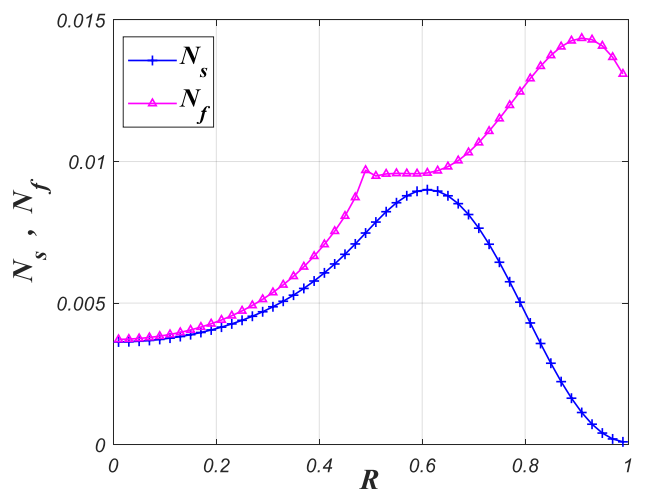

(b) Double-layer: $D a_{1}=10^{-3}, D a_{2}=10^{-2}$

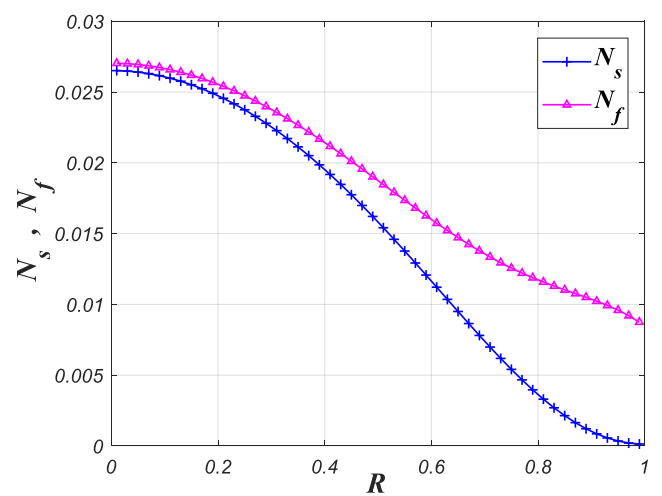

(d) Single layer: $D a=10^{-2}$

Figure 9. Local entropy generation rate varies with $R$ for different Darcy number $\left(\varepsilon_{1}=\varepsilon_{2}=0.9\right.$, $\left.B i_{1}=B i_{2}=0.1, k_{1}=k_{2}=0.01, k_{s 2}=1, F=50, P e=10, B r=10^{-4}, R_{1}=0.5\right)$.

Figure 10 represents the impact of the $k_{1}$ and $k_{2}$ on the local entropy generation rate. As shown in Figure 10, increasing the $k_{1}$ and $k_{2}$ sharply decreases the values of local entropy generation rates. Increasing the $k_{1}$ and $k_{2}$ decreases the thermal conductivity resistance and the temperature difference between two phases in porous media, which reduces the entropy generation caused by the heat conduction within the fluid phase and the heat exchange between two phases in porous media simultaneously.

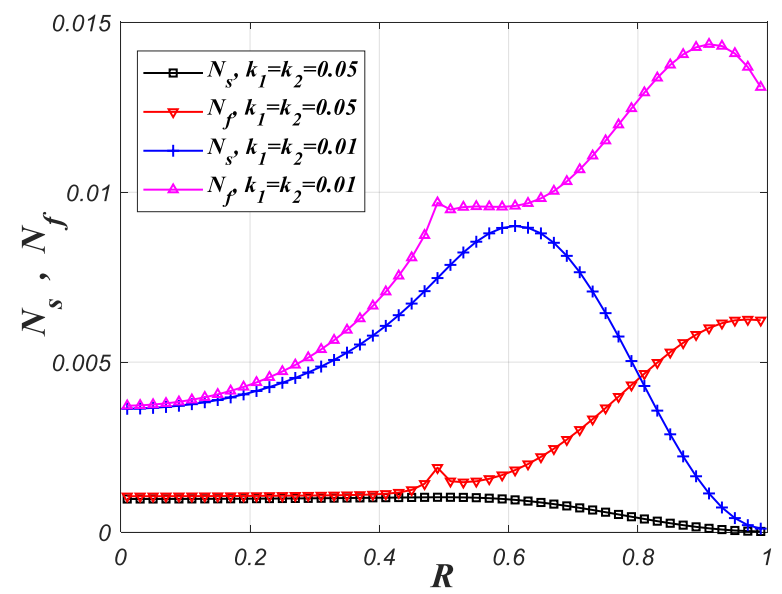

Figure 10. Local entropy generation rate varies with $R$ for different $k_{1}$ and $k_{2}\left(\varepsilon_{1}=\varepsilon_{2}=0.9\right.$, $\left.D a_{1}=10^{-3}, D a_{2}=10^{-2}, B i_{1}=B i_{2}=0.1, k_{s 2}=1, F=50, \mathrm{Pe}=10, \mathrm{Br}=10^{-4}, R_{1}=0.5\right)$. 
The effect of $D a_{1}$ on $N_{t}$ is shown in Figure 11. When the Biot number is small, as shown in Figure 11a, for the case of $R_{1}=0.5$, the $N_{t}$ increases with the increase of $D a_{1}$, for the case of $R_{1}=0.8$, the $N_{t}$ slightly decreases first and then increases with the increase of $D a_{1}$. Two effects will be induced by the increase of $D a_{1}$ : (a) the temperature difference between two phases in porous media will increase with the increase of $D a_{1}$, as shown in Figure 5, which will decrease the $N u$, as shown in Figure 7 a, and increase the entropy generation caused by heat exchange between two phases in porous media; and (b) the entropy generation caused by fluid friction will decrease with the increase of permeability. As a result, a minimum value can be found when $R_{1}=0.8$. When $R_{1}=0.5$, the proportion of the entropy generation caused by fluid friction is relatively small. Hence, the $N_{t}$ varies monotonically with $D a_{1}$. When the Biot number is large, the proportion of the entropy generation caused by heat transfer is relatively small, and the entropy generation caused by fluid friction will decrease with the increase of $D a_{1}$. Therefore, $N_{t}$ will decrease with the increase of $D a_{1}$, as shown in Figure $11 \mathrm{~b}$.

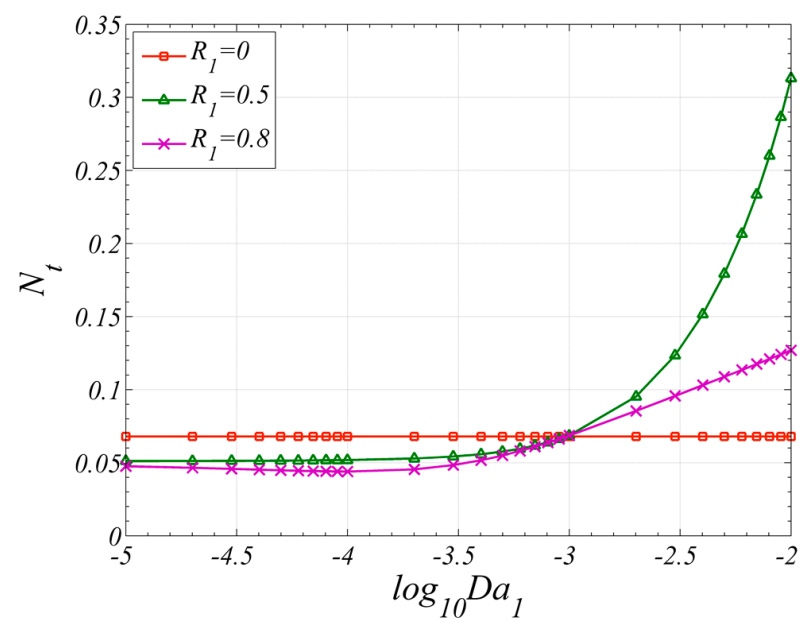

(a) $B i_{1}=B i_{2}=0.1$

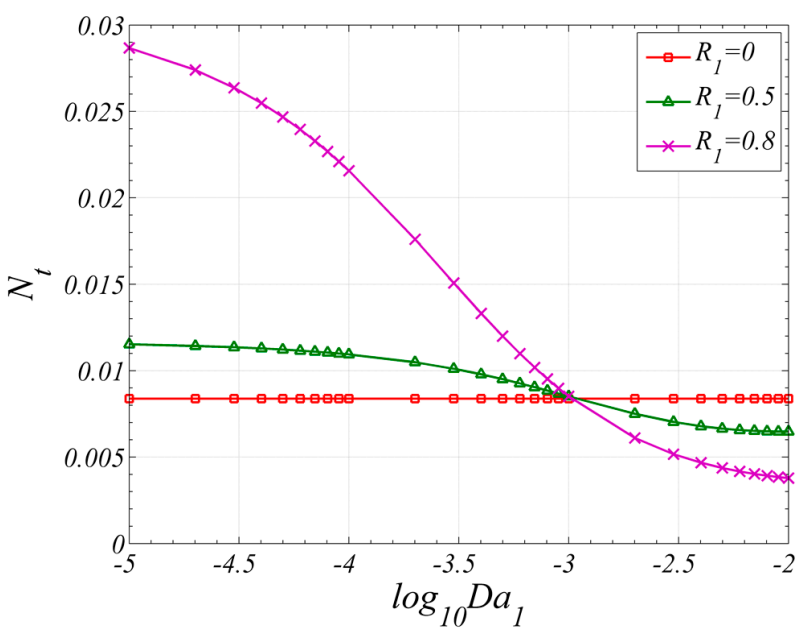

(b) $B i_{1}=B i_{2}=10$

Figure 11. $N_{t}$ varies with $D a_{1}$ for different $R_{1}$. $\left(\varepsilon_{1}=\varepsilon_{2}=0.9, D a_{2}=10^{-3}, k_{1}=k_{2}=0.01, k_{s 2}=1\right.$, $F=50, P e=10, B r=10^{-4}$ ).

Figure 12 shows the influence of $R_{1}$ on the $N_{t}$. When the Biot number is small, both the effects of the heat transfer and fluid friction should be considered. When $D a_{1}<D a_{2}$, as the result of the $N u$ variation with $R_{1}$, which is shown in Figure 7a, the entropy generation caused by heat transfer will decrease firstly and then increase with the increase of $R_{1}$. In the meantime, the entropy generation caused by fluid friction will increase with the increase of $R_{1}$. Therefore, it can be found in Figure 12a that there is 
a minimum value for $N_{t}$ which is smaller than that for the tube fully filled with corresponding single layer porous medium $\left(R_{1}=0\right.$, or $\left.R_{1}=1\right)$. When $D a_{1}>D a_{2}$, with the increase of $R_{1}$, the entropy generation caused by heat transfer will increase firstly and then decrease, and the entropy generation caused by fluid friction will decrease. Therefore, it can be found in Figure 12a that there is a maximum value for $N_{t}$.

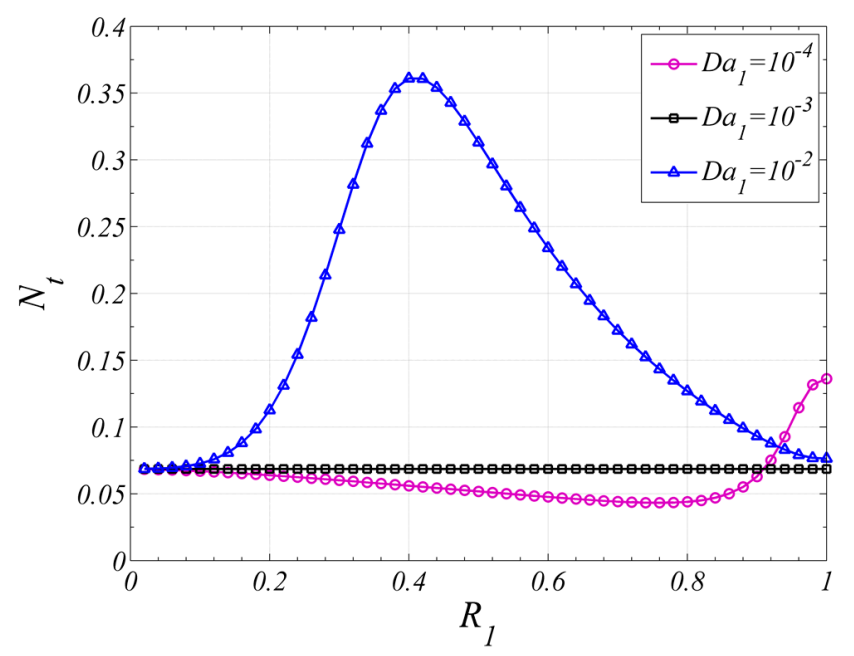

(a) $B i_{1}=B i_{2}=0.1$

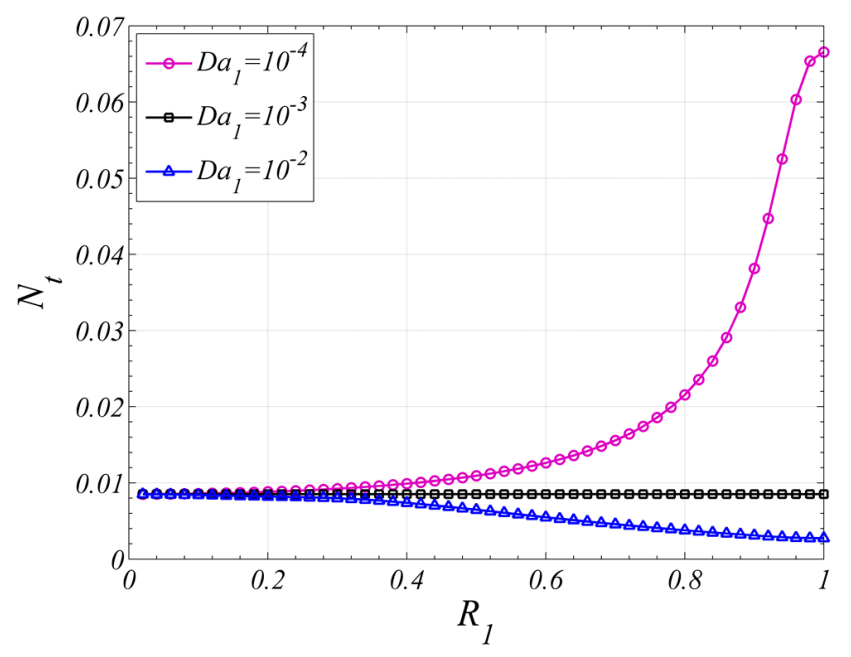

(b) $B i_{1}=B i_{2}=10$

Figure 12. $N_{t}$ varies with $R_{1}$ for different $D a_{1}\left(\varepsilon_{1}=\varepsilon_{2}=0.9, k_{1}=k_{2}=0.01, k_{s 2}=1, D a_{2}=10^{-3}\right.$, $F=50, P e=10, B r=10^{-4}$ ).

However, when the Biot number is relatively large, as shown in Figure 12b, for the case of $D a_{1}=10^{-4}$, the entropy generation is mainly caused by fluid friction; therefore, the $N_{t}$ increases with the increase of $R_{1}$. For the case of $D a_{1}=10^{-2}$, the entropy generation caused by both heat transfer and fluid friction are much small, and the $N_{t}$ decreases with the increase of $R_{1}$.

Figure 13 illustrates the influence of the $k_{s 2}$ on $N_{t}$. It can be found that both increasing the Biot number and increasing the $k_{s 2}$ can reduce the $N_{t}$. The influence of $k_{s 2}$ on $N_{t}$ is negligible when $k_{s 2}>1$. When the Biot number is small, as what have been mentioned above, a minimum value of $N_{t}$ can be found in Figure 13a. However, compared with Figures 7a and 12a, it can be found that the dimensionless interfacial radius corresponding to the maximum value of the $N u$ is different from that corresponding to the minimum value of the $N_{t}$. 


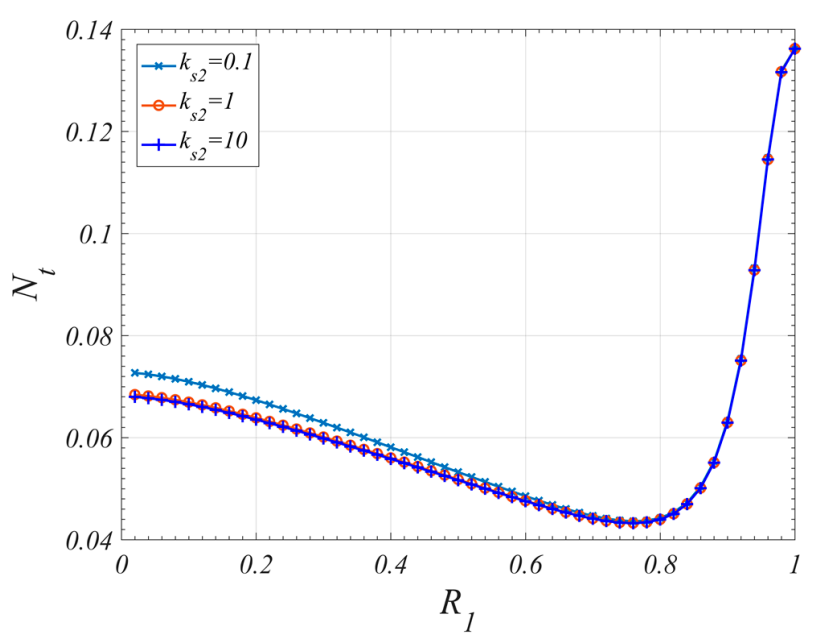

(a) $B i_{1}=B i_{2}=0.1$

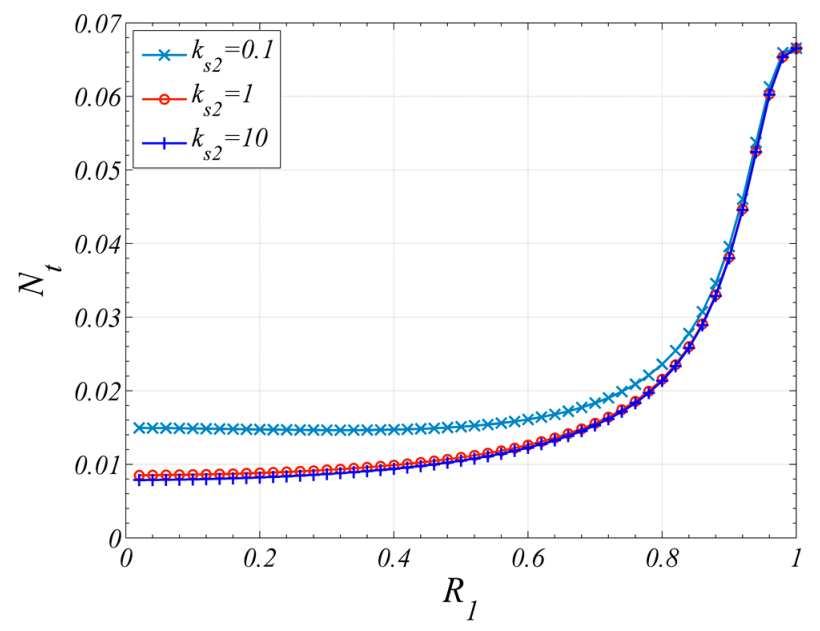

(b) $B i_{1}=B i_{2}=10$

Figure 13. $N_{t}$ varies with $R_{1}$ for different $k_{s 2}\left(\varepsilon_{1}=\varepsilon_{2}=0.9, D a_{1}=10^{-4}, D a_{2}=10^{-3}, k_{1}=k_{2}=0.01\right.$, $\left.F=50, \mathrm{Pe}=10, \mathrm{Br}=10^{-4}\right)$.

\section{Conclusions}

In this study, the forced convective heat transfer in a tube filled with double-layer porous media is analytically investigated. The Darcy-Brinkman equation and the LTNE model are employed for momentum and energy equations, respectively. The velocity field and temperature distributions are analytically solved, and the analytical solutions for Nusselt number, local, and total entropy generation rates are obtained. The analytical solutions are validated in the limiting case. Furthermore, the influences of the Darcy number, Biot number, thermal conductivity ratio, and dimensionless interfacial radius on flow and heat transfer, as well as irreversibility, are analyzed. The main conclusions are as follows:

(1) A more uniform temperature distribution of fluid phase within the tube filled with double-layer porous media can be obtained by decreasing $D a_{1}$ or increasing $D a_{2}$.

(2) When $D a_{1}$ is less than $D a_{2}$, the Nusselt number for the tube filled with double-layer porous media can be larger than that for the tube filled with corresponding single layer porous medium. However, when $D a_{1}$ is larger than $D a_{2}$, the Nusselt number for the tube filled with double-layer porous media can be less than that for the tube filled with corresponding single layer porous medium.

(3) When $D a_{1}$ is less than $D a_{2}$ and the Biot number is small, the total entropy generation rate for the tube filled with double-layer porous media can be less than that for the tube filled with corresponding single layer porous medium. 
(4) When $D a_{1}$ is less than $D a_{2}$, the maximum value of the Nusselt number and the minimum value of the total entropy generation rate for the tube filled with double-layer porous media can be obtained by properly selecting the pertinent parameters, such as Darcy number, Biot number, and dimensionless interfacial radius. However, it should be noted that the dimensionless interfacial radius corresponding to the maximum value of the Nusselt number is different from that corresponding to the minimum value of the total entropy generation rate.

Author Contributions: Conceptualization, K.Y. and J.W.; Data curation, W.H.; Formal analysis, K.Y. and W.H.; Investigation, K.Y. and W.H.; Methodology, K.Y. and W.H.; Resources, K.Y. and W.H.; Software, W.H.; Supervision, K.Y. and J.W.; Validation, W.H.; Writing—original draft, K.Y. and W.H.; Writing—review \& editing, K.Y. and X.L. All authors have read and agreed to the published version of the manuscript.

Funding: This work was supported by the National Natural Science Foundation of China (No. 51476063).

Conflicts of Interest: The authors declare no conflict of interest.

\section{Nomenclature}

a interfacial area per unit volume of the porous media, $\mathrm{m}^{-1}$

Bi Biot number

$\mathrm{Br} \quad$ Brinkman number

$c_{f} \quad$ heat capacity of fluid, $\mathrm{J} \cdot \mathrm{kg}^{-1} \cdot \mathrm{K}^{-1}$

$\mathrm{Da} \quad$ Darcy number

$F \quad$ dimensionless wall temperature, defined in Equation (15)

$h \quad$ heat transfer coefficient, $\mathrm{W} \cdot \mathrm{m}^{-2} \cdot \mathrm{K}^{-1}$

$K \quad$ permeability, $\mathrm{m}^{2}$

$k$ ratio of effective fluid thermal conductivity to that of solid

$k_{s} \quad$ ratio of effective solid thermal conductivity of two layers

$N_{f}^{\prime \prime \prime} \quad$ dimensionless local entropy generation rate within the fluid phase

$N_{s}^{\prime \prime \prime} \quad$ dimensionless local entropy generation rate within the solid phase

$N_{t} \quad$ dimensionless total entropy generation rate within the tube

$\mathrm{Nu} \quad$ overall Nusselt number

$P \quad$ dimensionless pressure drop

$P e \quad$ Peclet number

$p \quad$ pressure, $\mathrm{N} \cdot \mathrm{m}^{-2}$

$R \quad$ dimensionless radius

$r \quad$ radius, $\mathrm{m}$

$S_{S}^{\prime \prime \prime} \quad$ local entropy generation rate within the fluid phase

$S_{f}^{\prime \prime \prime} \quad$ local entropy generation rate within the solid phase

$T$ temperature, $\mathrm{K}$

$t k r$ thermal conductivity ratio, $k_{e} / k_{f}$

$U$ dimensionless velocity

$u \quad$ velocity, $\mathrm{m} / \mathrm{s}$

Greek symbols

$\varepsilon \quad$ porosity

$\theta \quad$ dimensionless temperature

$\mu \quad$ dynamic viscosity, $\mathrm{kg} \cdot \mathrm{m}^{-1} \cdot \mathrm{s}^{-1}$

$\rho \quad$ density, $\mathrm{kg} \cdot \mathrm{m}^{-3}$

Subscripts

b bulk

e effective

$f \quad$ fluid phase

$m$ mass average

$s$ solid phase

$w$ wall

1 layer 1

2 layer 2 
Appendix A . $\theta_{f, b}=Q_{1}-Q_{2}+Q_{3}-Q_{4}$

$$
\begin{aligned}
& Q_{1}=\frac{2 P^{2}}{k_{1}+k_{s 1}}\left\{\begin{array}{l}
{\left[C_{1} D_{1}+\frac{2 t_{1}^{2} D a_{1} C_{1}}{s_{1}^{2}\left(s_{1}^{2}-t_{1}^{2}\right)}-\frac{2 D a_{1} C_{1}}{t_{1}^{2}}\right] \times \frac{I_{1}\left(s_{1} R_{1}\right) R_{1}}{s_{1}}+} \\
C_{1} D_{3} \frac{I_{1}\left(s_{1} R_{1}\right) I_{0}\left(t_{1} R_{1}\right) s_{1} R_{1}-I_{0}\left(s_{1} R_{1}\right) I_{1}\left(t_{1} R_{1}\right) t_{1} R_{1}}{s_{1}^{2}-t_{1}^{2}} \\
-\frac{t_{1}^{2} C_{1}^{2} R_{1}^{2}}{s_{1}^{2}\left(s_{1}^{2}-t_{1}^{2}\right)}\left[I_{0}^{2}\left(s_{1} R_{1}\right)-I_{1}^{2}\left(s_{1} R_{1}\right)\right]- \\
\frac{D a_{1} C_{1}}{2}\left[\frac{I_{1}\left(s_{1} R_{1}\right) R_{1}^{3}}{s_{1}}-\frac{2 I_{0}\left(s_{1} R_{1}\right) R_{1}^{2}}{s_{1}^{2}}+\frac{4 I_{1}\left(s_{1} R_{1}\right) R_{1}}{s_{1}^{3}}\right]+ \\
\left(\frac{2 D a_{1}^{2}}{t_{1}^{2}}-D a_{1} D_{1}\right) \times \frac{R_{1}^{2}}{2}-D a_{1} D_{3} \times \frac{I_{1}\left(t_{1} R_{1}\right) R_{1}}{t_{1}}+\frac{D a_{1}^{2} R_{1}^{4}}{8}
\end{array}\right\} \\
& Q_{2}=\frac{2 P^{2}}{k_{1}}\left\{\begin{array}{l}
C_{1} D_{3} \frac{I_{1}\left(s_{1} R_{1}\right) I_{0}\left(t_{1} R_{1}\right) s_{1} R_{1}-I_{0}\left(s_{1} R_{1}\right) I_{1}\left(t_{1} R_{1}\right) t_{1} R_{1}}{s_{1}^{2}-t_{1}^{2}}-\frac{C_{1}^{2} R_{1}^{2}}{s_{1}^{2}-t_{1}^{2}}\left[I_{0}^{2}\left(s_{1} R_{1}\right)-I_{1}^{2}\left(s_{1} R_{1}\right)\right]+ \\
\left(\frac{2 D a_{1} C_{1}}{s_{1}^{2}-t_{1}^{2}}-\frac{2 D a_{1} C_{1}}{t_{1}^{2}}\right) \times \frac{I_{1}\left(s_{1} R_{1}\right) R_{1}}{s_{1}}-D a_{1} D_{3} \times \frac{I_{1}\left(t_{1} R_{1}\right) R_{1}}{t_{1}}+\frac{D a_{1}^{2} R_{1}^{2}}{t_{1}^{2}}
\end{array}\right\}
\end{aligned}
$$

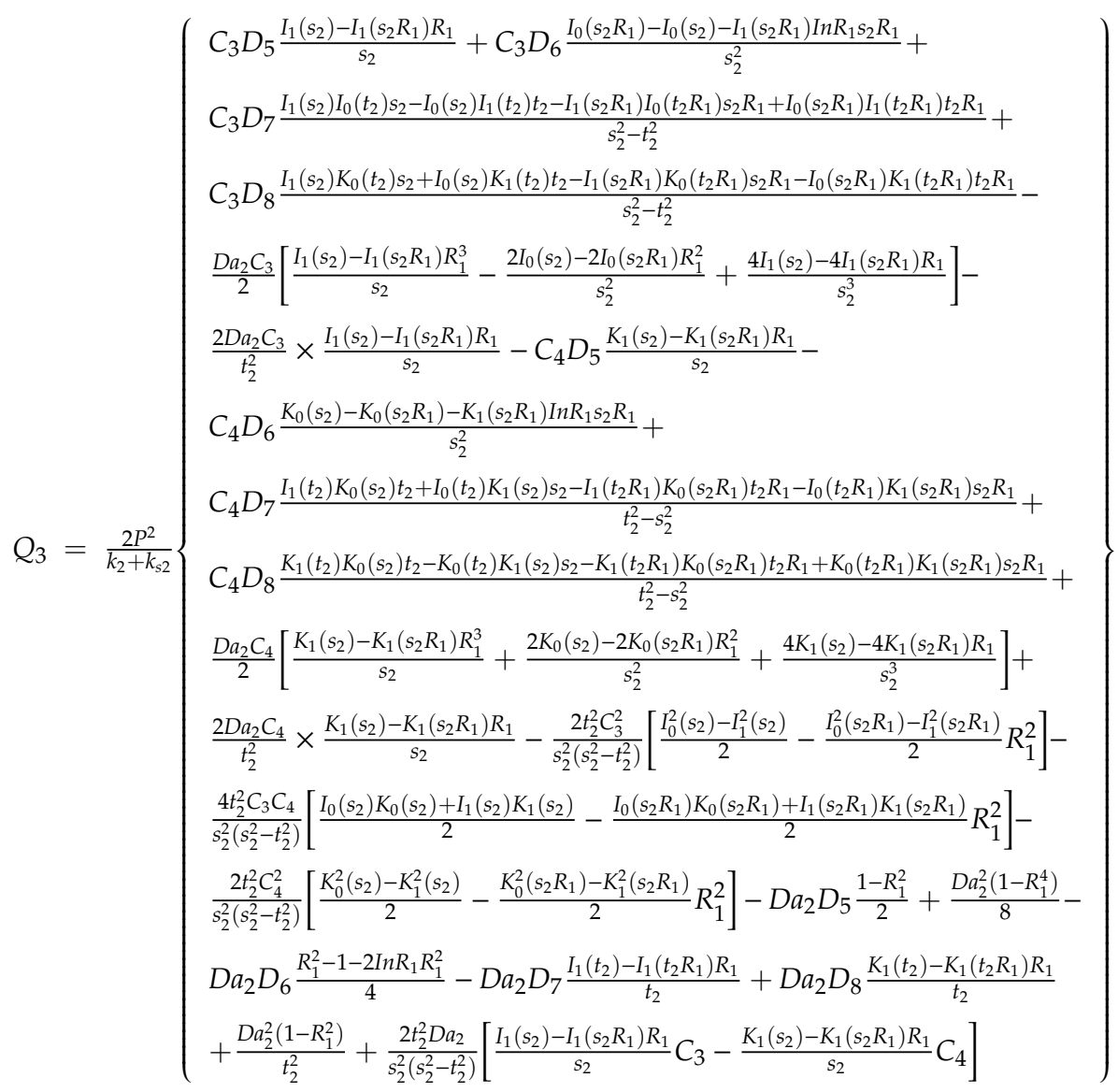




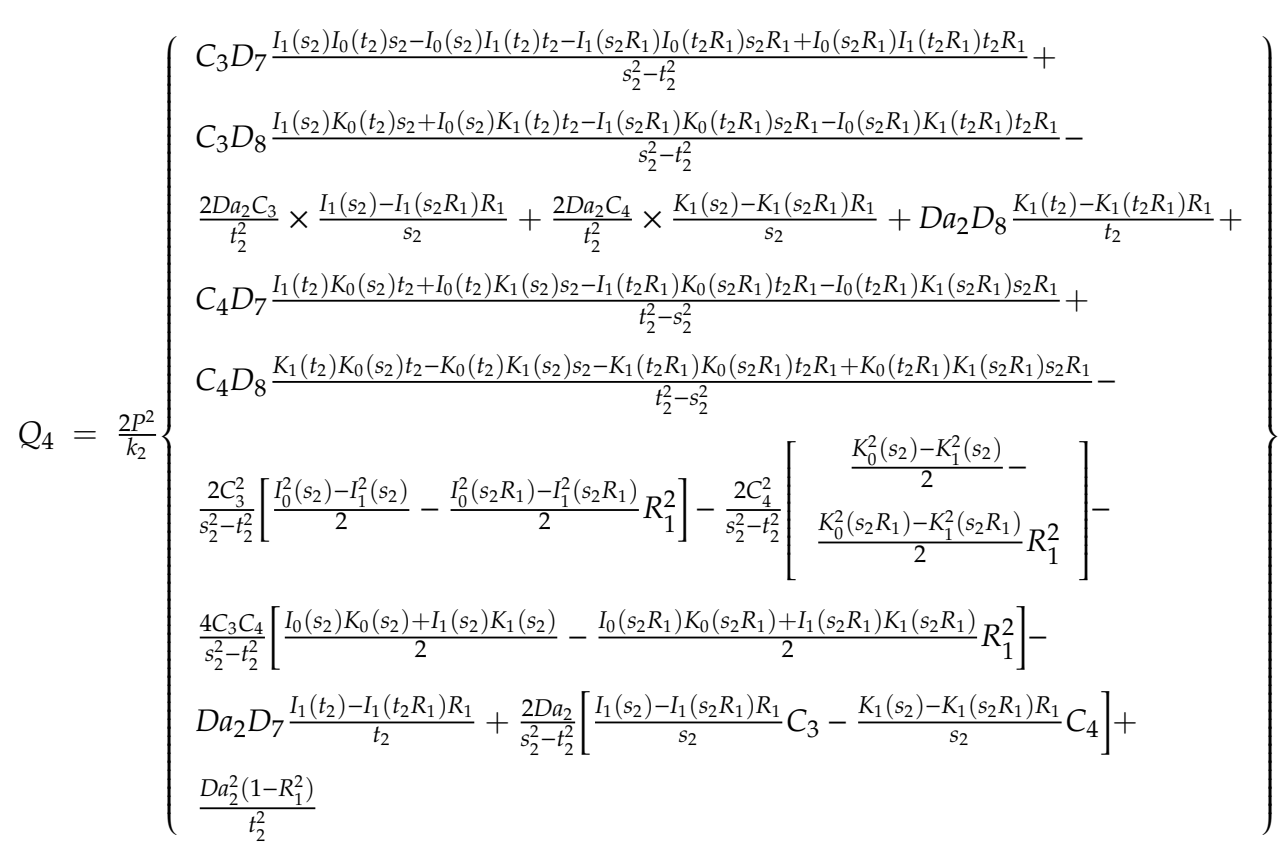

\section{References}

1. Kim, S.; Kim, D.; Lee, D. On the local thermal equilibrium in microchannel heat sinks. Int. J. Heat Mass Transf. 2000, 43, 1735-1748. [CrossRef]

2. Jing, D.; Song, J. Hydraulic and thermal performances of laminar flow in fractal treelike branching microchannel network with wall velocity slip. Fractals 2020, 28, 2050022. [CrossRef]

3. Jing, D.; Yi, S. Electroosmotic flow in tree-like branching microchannel network. Fractals 2019, $27,1950095$. [CrossRef]

4. Pavel, B.I.; Mohamad, A.A. Experimental Investigation of the Potential of Metallic Porous Inserts in Enhancing Forced Convective Heat Transfer. ASME J. Heat Transf. 2004, 126, 540-545. [CrossRef]

5. Lu, W.; Zhao, C.; Tassou, S. Thermal analysis on metal-foam filled heat exchangers. Part I: Metal-foam filled pipes. Int. J. Heat Mass Transf. 2006, 49, 27512761. [CrossRef]

6. Yang, K.; Vafai, K. Analysis of temperature gradient bifurcation in porous media-An exact solution. Int. J. Heat Mass Transf. 2010, 53, 4316-4325. [CrossRef]

7. Yang, K.; Vafai, K. Analysis of heat flux bifurcation inside porous media incorporating inertial and dispersion effects-An exact solution. Int. J. Heat Mass Transf. 2011, 54, 5286-5297. [CrossRef]

8. Xu, H.; Qu, Z.; Tao, W. Thermal transport analysis in parallel-plate channel filled with open-celled metallic foams. Int. J. Heat Mass Transf. 2011, 38, 868873. [CrossRef]

9. Dehghan, M.; Jamal-Abad, M.T.; Rashidi, S. Analytical interpretation of the local thermal non-equilibrium condition of porous media imbedded in tube heat exchangers. Energy Convers. Manag. 2014, 85, 264-271. [CrossRef]

10. Zhang, J.; Qu, Z.; Xu, H.; Tao, W. Semi-analytical solution for fully developed forced convection in metal-foam filled tube with uniform wall temperature. Sci. China Technol. Sci. 2014, 57, 2487-2499. [CrossRef]

11. Dehghan, M.; Valipour, M.S.; Saedodin, S. Temperature-dependent conductivity in forced convection of heat exchangers filled with porous media: A perturbation solution. Energy Convers. Manag. 2015, 91, 259-266. [CrossRef]

12. Yang, K.; You, X.; Wang, J.; Vafai, K. Analysis of two approaches for an adiabatic boundary condition in porous media. Int. J. Numer. Methods Heat Fluid Flow 2016, 26, 977-998. [CrossRef]

13. Torabi, M.; Karimi, N.; Zhang, K. Heat transfer and second law analyses of forced convection in a channel partially filled by porous media and featuring internal heat sources. Energy 2015, 93, 106-127. [CrossRef]

14. Torabi, M.; Karimi, N.; Zhang, K.; Peterson, G. Generation of entropy and forced convection of heat in a conduit partially filled with porous media-local thermal non-equilibrium and exothermicity effects. Appl. Therm. Eng. 2016, 106, 518-536. [CrossRef] 
15. Torabi, M.; Zhang, K.; Yang, G.; Wang, J.; Wu, P. Heat transfer and entropy generation analyses in a channel partially filled with porous media using local thermal non-equilibrium model. Energy 2015, 82, 922-938. [CrossRef]

16. Yang, K.; Chen, H.; Wang, J. Analysis of heat transfer and entropy generation in a channel partially filled with N-layer porous media. ASME J. Heat Transf. 2018, 140, 082601. [CrossRef]

17. Yang, K.; Vafai, K. Restrictions on the validity of the thermal conditions at the porous-fluid interface: An exact solution. ASME J. Heat Transf. 2011, 133, 112601. [CrossRef]

18. Mahmoudi, Y.; Karimi, N.; Mazaheri, K. Analytical investigation of heat transfer enhancement in a channel partially filled with porous material under local thermal non-equilibrium condition: Effect of different boundary conditions at porous fluid interface. Int. J. Heat Mass Transf. 2014, 70, 875-891. [CrossRef]

19. Mohamed, R.E.; Al-Hossainy, A.F. FEM for blood-based SWCNTs flow through a circular cylinder in a porous medium with electromagnetic radiation. Commun. Theor. Phys. 2019, 71, 1425.

20. Buonomo, B.; Manca, O.; Lauriat, G. Forced convection in micro-channels filled with porous media in local thermal non-equilibrium conditions. Int. J. Therm. Sci. 2014, 77, 206-222. [CrossRef]

21. Nield, D.A.; Kuznetsov, A.V. Effects of heterogeneity in forced convection in a porous medium: Parallel plate channel or circular duct. Int. J. Heat Mass Transf. 2000, 43, 4119-4134. [CrossRef]

22. Nield, D.A. Effects of heterogeneity in forced convection in a porous medium: Parallel-plate channel, asymmetric property variation, and asymmetric heating. J. Porous Media 2001, 4, 137148. [CrossRef]

23. Nield, D.A.; Kuznetsov, A.V. Effects of Temperature-Dependent Viscosity in Forced Convection in a Porous Medium: Layered-Medium Analysis. J. Porous Media 2003, 6, 213-222. [CrossRef]

24. Sundaravadivelu, K.; Tso, C.P. Influence of viscosity variations on the forced convection flow through two types of heterogeneous porous media with isoflux boundary condition. Int. J. Heat Mass Transf. 2003, 46, 2329-2339. [CrossRef]

25. Nield, D.A.; Kuznetsov, A.V. Interaction of Transverse Heterogeneity and Thermal Development of Forced Convection in a Porous Medium. Transp. Porous Media 2004, 57, 103-111. [CrossRef]

26. Nield, D.A.; Kuznetsov, A.V. Optimization of forced convection heat transfer in a composite porous medium channel. Transp. Porous Media 2013, 99, 349-357. [CrossRef]

27. Wildi-Tremblay, P.; Gosselin, L. Layered porous media architecture for maximal cooling. Int. J. Heat Mass Transf. 2007, 50, 464-478. [CrossRef]

28. Zheng, Z.J.; Li, M.J.; He, Y.L. Optimization of porous insert configurations for heat transfer enhancement in tubes based on genetic algorithm and CFD. Int. J. Heat Mass Transf. 2015, 87, 376-379. [CrossRef]

29. Chu, L.; Fwa, T.F. Functional sustainability of single- and double-layer porous asphalt pavements. Constr. Build. Mater. 2019, 197, 436-443. [CrossRef]

30. Misra, J.C.; Mallick, B.; Steinmann, P. Temperature distribution and entropy generation during Darcy-Forchheimer-Brinkman electrokinetic flow in a microfluidic tube subject to a prescribed heat flux. Meccanica 2020, 55, 1079-1098. [CrossRef]

31. Nield, D.A. Effects of heterogeneity in forced convection in a porous medium: Parallel-plate channel, Brinkman model. J. Porous Media 2003, 6, 257-266. [CrossRef]

32. Nield, D.A.; Kuznetsov, A.V. The interaction of thermal nonequilibrium and heterogeneous conductivity effects in forced convection in layered porous channels. Int. J. Heat Mass Transf. 2001, 44, 4369-4373. [CrossRef]

33. Xu, H.; Qu, Z.; Tao, W. Analytical solution of forced convective heat transfer in tubes partially filled with metallic foam using the two-equation model. Int. J. Heat Mass Transf. 2011, 54, 3846-3855. [CrossRef]

34. Allan, F.M.; Hamdan, M.H. Fluid Mechanics of the Interface Region between Two Porous Layers. Appl. Math. Comput. 2002, 128, 37-43. [CrossRef]

35. Vafai, K.; Thiyagaraja, R. Analysis of Flow and Heat Transfer at the Interface Region of a Porous Medium. Int. J. Heat Mass Transf. 1987, 30, 1391-1405. [CrossRef]

36. Mahjoob, S.; Vafai, K. Analysis of Bioheat Transport Through a Dual Layer Biological Media. ASME J. Heat Transf. 2010, 132, 031101. [CrossRef]

37. Zwillinger, D. CRC Standard Mathematical Tables and Formulae, 32nd ed.; CRC Press: New York, NY, USA, 2011; pp. 454-462.

38. Bejan, A. Entropy Generation through Heat and Fluid Flow; John Wiley \& Sons: New York, NY, USA, 1982; pp. 98-105. 
39. Pavel, B.I.; Mohamad, A.A. An experimental and numerical study on heat transfer enhancement for gas heat exchangers fitted with porous media. Int. J. Heat Mass Transf. 2004, 47, 4939-4952. [CrossRef]

Publisher's Note: MDPI stays neutral with regard to jurisdictional claims in published maps and institutional affiliations.

(C) 2020 by the authors. Licensee MDPI, Basel, Switzerland. This article is an open access article distributed under the terms and conditions of the Creative Commons Attribution (CC BY) license (http://creativecommons.org/licenses/by/4.0/). 